\title{
A survey of RNA secondary structural propensity encoded within human herpesvirus genomes: global comparisons and local motifs
}

\author{
Ryan J. Andrews ${ }^{1}$, Collin A. O'Leary ${ }^{1}$, Walter N. Moss ${ }^{\text {Corresp. } 1}$ \\ ${ }^{1}$ The Roy J. Carver Department of Biochemistry, Biophysics and Molecular Biology, lowa State University, Ames, IA, US \\ Corresponding Author: Walter N. Moss \\ Email address: wmoss@iastate.edu
}

There are 9 herpesviruses known to infect humans, of which Epstein-Barr virus (EBV) is the most widely distributed ( $>90 \%$ of adults infected). This ubiquitous virus is implicated in a variety of cancers and autoimmune diseases. Previous analyses of the EBV genome revealed numerous regions with evidence of generating unusually stable and conserved RNA secondary structures and led to the discovery of a novel class of EBV non-coding (nc)RNAs: the stable intronic sequence (sis)RNAs. To gain a better understanding of the roles of RNA structure in EBV biology and pathogenicity, we revisit EBV using recently developed tools for genome-wide motif discovery and RNA structural characterization. This corroborated previous results and revealed novel motifs with potential functionality; one of which has been experimentally validated. Additionally, since many herpesviruses increasingly rival the seroprevalence of EBV (VZV, HHV-6 and HHV-7 being the most notable), analyses were expanded to include all sequenced human Herpesvirus RefSeq genomes, allowing for genomic comparisons. In total 10 genomes were analyzed, for EBV (types 1 and 2), HCMV, HHV-6A, HHV-6B, HHV-7, HSV-1, HSV-2, KSHV, and VZV. All resulting data were archived in the RNAStructuromeDB ( https://structurome.bb.iastate.edu/herpesvirus ) to make them available to a wide array of researchers. 


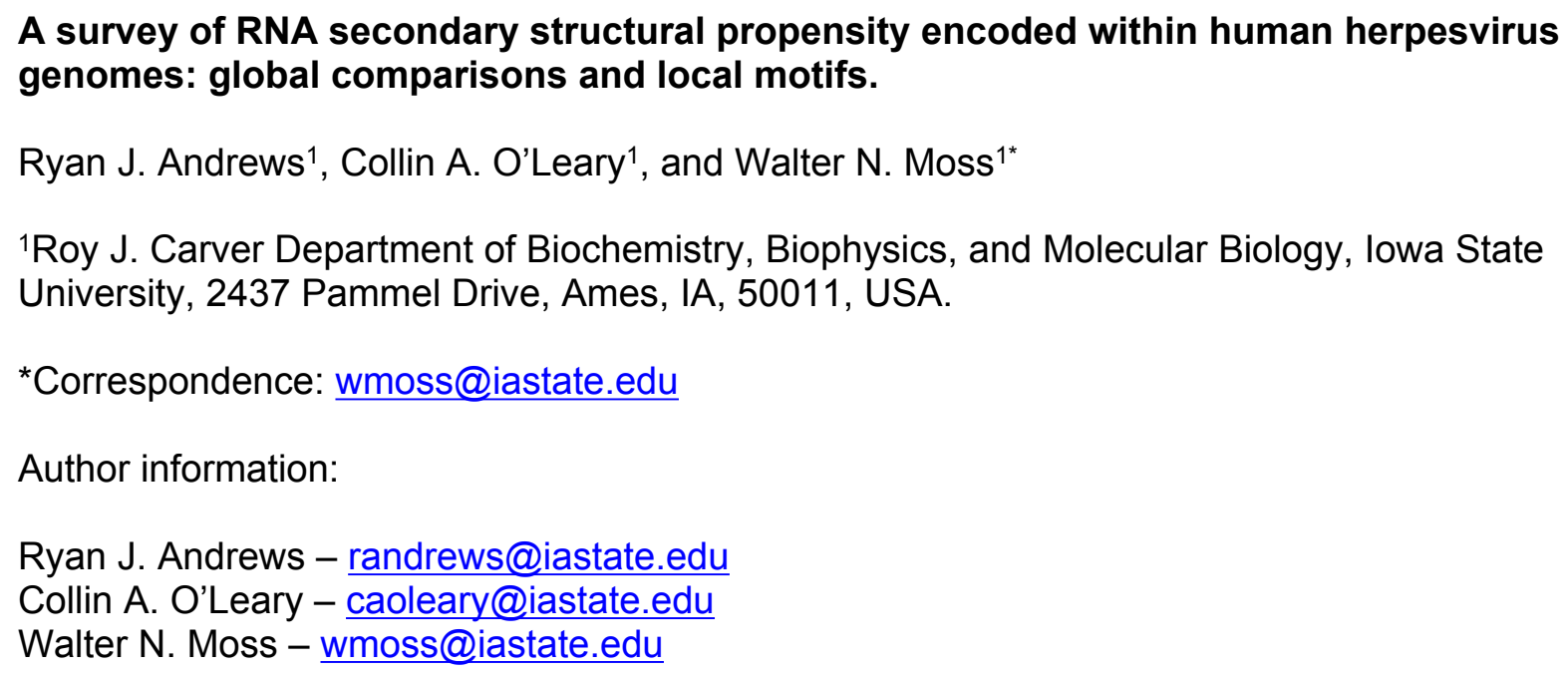




\section{Abstract}

There are 9 herpesviruses known to infect humans, of which Epstein-Barr virus (EBV) is the most widely distributed ( $>90 \%$ of adults infected). This ubiquitous virus is implicated in a variety of cancers and autoimmune diseases. Previous analyses of the EBV genome revealed numerous regions with evidence of generating unusually stable and conserved RNA secondary structures and led to the discovery of a novel class of EBV non-coding (nc)RNAs: the stable intronic sequence (sis)RNAs. To gain a better understanding of the roles of RNA structure in EBV biology and pathogenicity, we revisit EBV using recently developed tools for genome-wide motif discovery and RNA structural characterization. This corroborated previous results and revealed novel motifs with potential functionality; one of which has been experimentally validated. Additionally, since many herpesviruses increasingly rival the seroprevalence of EBV (VZV, HHV-6 and HHV-7 being the most notable), analyses were expanded to include all sequenced human Herpesvirus RefSeq genomes, allowing for genomic comparisons. In total 10 genomes were analyzed, for EBV (types 1 and 2), HCMV, HHV-6A, HHV-6B, HHV-7, HSV-1, HSV-2, KSHV, and VZV. All resulting data were archived in the RNAStructuromeDB (https://structurome.bb.iastate.edu/herpesvirus) to make them available to a wide array of researchers.

\section{Introduction}

The herpesviruses are an ancient family of double-stranded DNA viruses (Order Herpesvirales, Family Herpesviridae). The three major subfamilies (clades) of mammalian herpesviruses- $\alpha$ (Alphaherpesvirinae), $\beta$ (Betaherpesvirinae), and $\mathrm{y}$ (Gammaherpesvirinae) -appeared $\sim 180$ to 220 mya(McGeoch et al. 1995). Nine herpesviruses are currently known to infect humans: Epstein-Barr virus (EBV), human cytomegalovirus (HCMV), human herpesvirus $6 \mathrm{~A}$ and $6 \mathrm{~B}$ (HHV-6A and HHV-6B), human herpesvirus 7 (HHV-7), herpes simplex viruses 1 and 2 (HSV-1 and HSV-2), varicella-zoster virus (VZV), and Kaposi's sarcoma-associated herpesvirus (KSHV). Of these, EBV is most prevalent with $>90 \%$ of adults being infected (Kieff \& Rickinson 2001). EBV has a large $(\sim 172 \mathrm{~kb})$ double-stranded DNA genome that recapitulates many of the biological processes important to the host genome (Young \& Rickinson 2004) including periods of pervasive transcription (O'Grady et al. 2014). After spreading through the host by lytic replication, EBV establishes latency in B cells via several distinct patterns of coding and noncoding gene expression. Both lytic and latent replication are associated with various cancers (e.g. Iymphomas and carcinomas) (Elgui de Oliveira et al. 2016) and autoimmune diseases (e.g. multiple sclerosis and Lupus) (Draborg et al. 2013); however, molecular mechanisms connecting EBV infection to disease remain elusive-making EBV an important target of study. One route for the regulation of viral/host biology and for mediating host-virus interactions is RNA secondary structure. Many key biological processes of both hosts and their pathogens are affected by RNA structure: e.g. RNA transcription, processing, stability, and expression are all affected by RNA folding (Andrews \& Moss 2019a; Bevilacqua et al. 2016).

Although several functional RNA structural motifs have been identified in EBV (Moss et al. 2014), knowledge of the roles of RNA and RNA folding in EBV infection and disease still require additional attention. The first viral noncoding (nc)RNAs, discovered in 1981, were the EBV encoded RNAs (EBERs -1 and -2)(Lerner et al. 1981). Both EBERs are highly-expressed in infected cells (dwarfing endogenous ncRNA expression) and possess extensive secondary structure that mediates their interactions with host and viral biomolecules to promote infection (Lee et al. 2015; Lee et al. 2012; Lee et al. 2016; Moss et al. 2014). Over 20 years later 
101

102

103

104

105

106

107

108

109

110

111

112

113

114

115

116

117

118

119

120

121

122

123

124

125

126

127

128

129

130

131

132

133

134

135

136

137

138

139

140

141

142

143

144

145

146

147

148

149

150

additional viral ncRNAs were discovered: the BART and BHRF EBV-encoded micro (mi)RNAs in 2004 (Pfeffer et al. 2004) and the EBV viral small nucleolar ( $v$-snoRNA)1 in 2009 (Hutzinger et al. 2009).

A genome-wide bioinformatics and experimental analysis was previously performed in EBV to scan for functional RNA motifs (Moss \& Steitz 2013). In the previous analysis, the ncRNA discovery program RNAz (Gruber et al. 2010) was used to analyze sliding windows of aligned EBV genome sequences (plus one closely related lymphocryptovirus from rhesus monkeys: Macacine herpesvirus 4) to predict windows that have unusual thermodynamic stability and conservation of RNA secondary structure-both indicators of functionality (Clote et al. 2005). Approximately $30 \%$ of the $(\sim 170 \mathrm{kbp})$ genome was predicted to potentially encode functional RNA structure. In addition to known structures (EBERs, v-snoRNA1, and pre-miRNA hairpins), many novel structural elements were predicted. This analysis discovered a new class of EBV ncRNAs, the stable intronic sequence (sis)RNAs, which consist of abundant, highly structured intronic-sequence RNAs (derived from the EBV W repeats). The sisRNAs consist of a complex mix of excised introns and, more recently discovered, alternatively spliced long (I)ncRNAs (up to $\sim 22 \mathrm{~kb}$ ) (Cao et al. 2015b; Tompkins et al. 2018). The sisRNAs interact with multiple host regulatory proteins (Tompkins et al. 2018) and are essential to EBV's ability to transform human $\mathrm{B}$ cells (Bridges et al. 2019). Very long ( 500 nt) hairpins were modeled in the latent origin of replication (oriP), which were later shown to occur in bidirectional lytic IncRNAs that are hyper A-to-I edited and promote lytic replication (Cao et al. 2015a). Elsewhere, a structural motif partially spans two exons and a whole intron in the EBV LMP2 gene; structure in this motif mediates interactions with splicing regulatory proteins and, surprisingly, nuclear actin to affect LMP2 splicing (a novel role for actin) (Kumarasinghe \& Moss 2019).

Although this analysis of EBV led to interesting discoveries, there were several limitations to this previous approach: (1) motifs that were not conserved (e.g. motifs unique to EBV) were potentially missed; (2) multiple alternative models were predicted for the same nt in different analysis windows; and (3) subjectively selected regions, comprised of overlapping windows with favorable metrics, were selected for generating final models. To address this, in this current study we used a new computational pipeline for RNA secondary structure analysis, ScanFold, which addresses these limitations and has been successfully applied to the Zika and HIV-1 genomes (Andrews et al. 2018) as well as the human MYC mRNA (O'Leary et al. 2019). The ScanFold pipeline divides RNA structural motif discovery into two parts; the first step is accomplished using ScanFold-Scan, where individual sequences are analyzed using a sliding window approach. This generates overlapping windows covering each nt of the sequence. For each window ScanFold-Scan calculates 4 folding metrics: (1) The minimum free energy

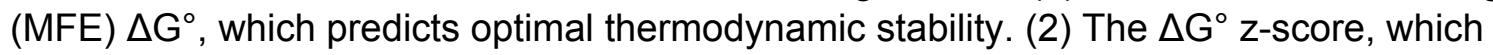
measures the stability of native vs. random sequence. (3) $\mathrm{A} p$-value for the $\Delta \mathrm{G}^{\circ} \mathrm{z}$-score, which is a quality control metric that calculates the fraction of randomized sequences with greater stability than native: $p$-values closer to 0 indicate a high confidence $z$-score. (4) The ED (ensemble diversity), which indicates the structural diversity of Boltzmann-weighted conformations calculated from a partition function. The second step, carried out by the program ScanFold-Fold, builds secondary structural models from base pairs likely to be functionally significant. ScanFold-Fold first compiles and averages ScanFold-Scan metrics for every base pairing arrangement (for every nucleotide) predicted across the sequence. The key metric used for selecting the most favorable bp for a nt is the $\Delta G^{\circ} z$-score ${ }_{\text {avg, }}$, which highlights bp that are consistently found in low z-score windows. This process was able to detect (and model with accuracy) all known structured motifs in the HIV-1 and ZIKV genomes (Andrews et al. 2018). Further analysis revealed the entirety of ScanFold-Fold results for HIV, ZIKV, and Hepatitis C

Peer) reviewing PDF | (2020:04:47551:1:1:NEW 5 Aug 2020) 
151

152

153

154

155

156

157

158

159

160

161

162

163

164

165

166

167

168

169

170

171

172

173

174

175

176

177

178

179

180

181

182

183

184

185

186

187

188

189

190

191

192

193

194

195

196

197

198

199

200

genomes were consistent with experimentally derived models - where the models from regions with the most negative $z$-score ${ }_{\text {avg }}$ values matched experimentally derived predictions and models in positive $z$-score ${ }_{\text {avg }}$ regions correlated to unstructured regions (Andrews et al. 2019). These results showed that $\mathrm{ScanFold}$ can serve not only as a motif discovery pipeline (Andrews et al. 2018), but as a tool to generally characterize RNA structural landscapes (Andrews et al. 2019).

In this current study we revisit EBV using this new approach, finding evidence for additional functional elements in EBV including one that was experimentally characterized. Significantly, in this new study, focus is placed on the more prevalent EBV type 1 RefSeq genome (Smatti et al. 2018) (rather than the type 2 genome previously analyzed); this current study, however, also includes data for the type 2 genome. Additionally, all sequenced human Herpesvirus RefSeq genomes have been analyzed to allow for global comparisons between genomes and to facilitate the identification of specific viral RNA motifs for further structure/function analyses. All results for EBV and other human herpesviruses are archived on the RNAStructuromeDB a public resource archiving RNA secondary structural data for humans and our pathogens (Andrews et al. 2017).

\section{Methods}

Human herpesvirus genome sequences were acquired from the NCBI RefSeq database (O'Leary et al. 2016): EBV-1 (NC_007605.1), EBV-2 (NC_009334.1), HCMV (NC_006273.2), HHV-6A (NC_001664.4), HHV-6B (NC_000898.1), HHV-7 (NC_001716.2), HSV-1 (NC_001806.2), HSV-2 (NC_001798.2), KSHV (NC_009333.1), VZV (NC_001348.1). Each sequence was analyzed using the $\mathrm{ScanFold}$ pipeline. First, genome sequences were scanned using a sliding analysis window of $150 \mathrm{nt}$ with a single nt step size using ScanFold-Scan. In each analysis window, the minimum Gibbs free energy of folding $(\Delta G)$ and its associated secondary structure were predicted, assuming the genome sequence was transcribed into RNA. A $\Delta \mathrm{G} z$-score is calculated by comparing the native sequence to the average $\Delta \mathrm{G}$ of 50 randomized sequences (shuffling nucleotides) according to the following equation: $z$-score $=$ $\left(\Delta G_{\text {native }}-\Delta G_{\text {random, average }}\right) / \sigma$; here, $\sigma$ is the standard deviation of all calculated $\Delta G$ s. Negative zscores indicate the number of standard deviations more stable the native sequence is vs. random. A p-value is calculated, which is a quality control metric for the z-score that measures the fraction of random sequences more stable than native (ideally, this will be close to zero). The ensemble diversity is calculated from a partition function, which measures the diversity of folds in the possible folding ensemble. Here, low numbers indicate that there is a single dominant conformation, while higher numbers indicate alternative conformations or a lack of stable structure. From the partition function a centroid structure is also calculated, this is the secondary structure model that has the least difference with all other Boltzmann weighted conformations in the ensemble-thus, it can be considered as a structure that represents the ensemble fold. The parameters used for window/step size and randomizations were previously optimized (Andrews et al. 2018).

The output of ScanFold-Scan were then analyzed using the program ScanFold-Fold. Here, unique motifs are generated by creating consensus secondary structures across all overlapping scanning windows, where base pairs are weighted by the z-score of the window in which they occur. Motifs are thus generated from the base pairs that most contribute to the unusual structural stability of a sequence. Presumably, the evolved order of these sequences creates the particular stability of these pairs, indicating their potential for function (e.g. evolution working to stabilize such structures). A key component of ScanFold-Fold is the decision-making 
201

202

203

204

205

206

207

208

209

210

211

212

213

214

215

216

217

218

219

220

221

222

223

224

225

226

227

228

229

230

231

232

233

234

235

236

237

238

239

240

241

242

243

244

245

246

247

248

249

250 process used to define base pairs when multiple, unusually stable, pairs can be predicted for a residue; here, the algorithm selects the pairing partner that most consistently contributed to low z-score windows throughout the scan (see Methods of (Andrews et al. 2018)). For regions of interest, these unusually stable base pairs were used as constraints for generating global secondary structural models. This was done to "fill in" base pairs that were "missing" due to the generation of the consensus structure (e.g. where multiple, equally stable, pairs were averaged out) and to deduce potential long-range interactions, which spanned regions larger than the (150 nt) windows used. To do this, the program RNA fold (Lorenz et al. 2011) was used to predict the minimum free energy secondary structure possible, given the constraint of forming all ScanFold-Fold with average z-score bp $<-1$ or -2 (described as the "Global Refold" option in (Andrews et al. 2019)).

To test predicted models for these select regions, a comparative sequence/structure analysis was performed. The EBV-1 sequence was used in a BLASTn analysis to deduce homologous sequence. These were aligned using the program MAFFT (Katoh et al. 2009; Katoh \& Frith 2012; Katoh et al. 2005; Katoh et al. 2002; Katoh et al. 2017; Katoh \& Standley 2013; Katoh \& Standley 2016; Katoh \& Toh 2008a; Katoh \& Toh 2008b; Katoh \& Toh 2010; Kuraku et al. 2013; Nakamura et al. 2018; Yamada et al. 2016) implementing the G-INS-i strategy, which is optimized for sequences with global homology. The ScanFold-Fold constrained secondary structure model for EBV-1 was mapped to this structure and conservation of base pairing was assessed as the percentage of canonical pairing (GC/CG, AU/UA, or GU/UG base pairs) observed across aligned positions. Mutations were tracked across model base pairs to identify structure-preserving mutations that were potentially consistent (single point mutations) or compensatory (double point mutations) with respect to the structure model.

To experimentally test RNA structural motifs for their effects on post-transcriptional gene regulation, select sequences were inserted into the pIS2 vector downstream of its Renilla Luciferase (RL) gene (within the 3' UTR). All conditions were tested in biological triplicate as described in (O'Leary et al. 2019). Briefly, RL constructs, along with Firefly (FF) Luciferase were transfected into HeLa cells and incubated at $37^{\circ} \mathrm{C}$ and $5 \% \mathrm{CO}_{2}$ while maintained in DMEM supplemented with 10\% FBS, penicillin, streptomycin, and L-glutamine. After $24 \mathrm{~h}$, transfected cells were split between a 24 well plate (for qPCR) and a 96 well plate (for luciferase assays) and incubated an additional $24 \mathrm{~h}$ before analysis. Dual luciferase assays were designed and performed following recommendations of (Van Etten et al. 2013): cells were lysed, and luciferase activity was measured using Promega's Dual Luciferase Reagent Assay kit with a collection time of 10 seconds. Relative response ratios (RRR), calculated as the ratio of RL to FF relative light units (RLUs), were calculated for each sample (Table S2).

The RT-qPCR analysis was carried out as described in (O'Leary et al. 2019): briefly, whole cell RNA was extracted in TRIzol followed by a Dnase I treatment (NEB) for $2 \mathrm{~h}$ at $37^{\circ} \mathrm{C}$. RNA was further purified with Zymo's RNA Clean and Concentrator kit before reverse transcription which was carried out using $1 \mu \mathrm{g}$ of purified RNA, random hexamers, and Superscript III (ThermoFisher). Transcript abundance was then measured via qPCR and analyzed using the $\Delta \Delta \mathrm{Ct}$ method, where the relative abundance of $\mathrm{RL}$ was calculated using FF as the reference gene. The results of both RT-qPCR and dual luciferase assays can be seen in Table S2.

\section{Results}

Global results 
251

252

253

254

255

256

257

258

259

260

261

262

263

264

265

266

267

268

269

270

271

272

273

274

275

276

277

278

279

280

281

282

283

284

285

286

287

288

289

290

291

292

293

294

295

296

297

298

299

300

Average MFE $\triangle G$ values across each genome ranged from $-24.8 \mathrm{kcal} / \mathrm{mol}(\mathrm{HHV}-7)$ to -64.1 $\mathrm{kcal} / \mathrm{mol}$ (HSV-2; Table 1). This large difference in predicted RNA folding stability, as one would expect, correlates with the GC\%, which ranged from $36.2 \%$ to $70.4 \%$. The average $z$-score and ensemble diversity (ED), however, do not follow trends for $\Delta \mathrm{G}$ or GC\%. The z-score quantifies the greater-than-random folding stability of an RNA sequence and is primarily dependent on the sequence order and not its composition. Likewise, the ED value indicates the diversity of potential structural conformations in an RNA's folding ensemble, which also appears to be an evolved property of ordered/functional RNA sequences (Moss 2018a). The average z-score ranged from -0.09 (HHV-6B) to -0.63 for EBV-1, while the average ED ranged from 33.7 (HHV7 ) to 32.3 (EBV-1). Indeed, all folding metrics suggested functional structured motifs were most prevalent in the EBV type 1 genome.

Unsurprisingly, the predictions for EBV-1 and EBV-2 were almost identical (Table 1), as the two genomes are only $\sim 5 \%$ different in sequence and the majority of this difference clusters within one gene region (EBNA-2) (Tzellos \& Farrell 2012). The percentage of the EBV genome spanned by windows with z-scores < -1 (one standard deviation more stable than random) was predicted to be $35.1 \%$ and $34.4 \%$ for types 1 and 2, respectively. This is consistent with previous predictions using RNAz, which predicted $\sim 30 \%$ of the genome to fall within low z-score windows (Moss \& Steitz 2013). These values are stronger than any other human herpesvirus genome: e.g. the next highest percentage of windows with z-score <-1 were for KSHV $(25 \%)$. This also held true for windows with z-scores < -2 (two standard deviations more stable than random), where EBV-1 and EBV-2 had $13.2 \%$ and $12.7 \%$ of their windows below this stricter cutoff: the next highest percentage was for HSV-1 (8.1\%). The virus with the smallest fraction of its genome spanned by low z-score windows was HHV-6B (19.0\% and $4.7 \%$ for the -1 and -2 zscore cutoffs, respectively).

In the prediction of the MFE $\Delta G$ for each analysis window, a model secondary structure is also generated. Across each genome this resulted in many predicted base pairs (e.g. the HCMV genome has 1,134,919 predicted base pairs; Table 1), where a specific nucleotide can be paired differently across several overlapping windows. As a result, many potential base pairing partners may be predicted per nucleotide, resulting in the total number of predicted base pairs to often exceed genome length; a confounding factor which has proved to be a challenge in RNA 2D structure modeling (see Introduction). The ScanFold-Fold algorithm confronts this challenge by predicting the single most likely orientation for each nucleotide based on its contributions to low z-score windows (indicating ordered stability and, potentially, function). The viruses that had the greatest percentages of low z-score base pairs were EBV-1 and EBV-2: EBV-1 had $8.8 \%$ of its base pairs predicted with average $z$-score $<-1$ and EBV-2 had $2.6 \%$ of its base pairs predicted with average z-score <-2 (Table 1). Additionally, ScanFold-Fold extracts each discrete structural motif (i.e. single hairpins or multi-branched stem loops) containing at least one base pair with an average z-score < -2. This results in a list of motifs for each genome, where again, EBV-1 and EBV-2 had the greatest number of motifs with 858 and 870 respectively.

In summary, all predictions indicate a particular importance for functional RNA secondary structures encoded within the EBV genome vs. other herpesviruses. The ScanFold-Fold results also give us a means of generating motifs of interest for further analysis.

Known structural motifs in EBV

Peer) reviewing PDF | (2020:04:47551:1:1:NEW 5 Aug 2020) 
301

The ScanFold analysis was able to deduce the location and, in many cases, the secondary structure of known RNA structural motifs in EBV-1. All known EBV-1 miRNA sequences fell within low z-score regions and were contained within hairpins formed by low z-score base pairs. For example, Figure 1 summarizes ScanFold results for the EBV-1 BART miRNA gene cluster. All but one miRNA sequence fall within hairpins comprised of base pairs with average z-score < -2 (Fig. 1a; ebv-BART-5 miRNAs occurred in a hairpin comprised of z-score < -1 base pairs). Features of the ScanFold-Fold model secondary structures for the EBV miRNAs are consistent with what is known about active precursor (pre)-miRNA hairpins. For example, the ebv-BART3* and ebv-BART3 sequences are annealed to each other, are offset by two nucleotides and contain internal bulge loops (Fig. 1b). A similarly sized hairpin was detected elsewhere in the EBV genome, which corresponds to the EBV viral small nucleolar (v-sno)RNA1 (Fig. 2) (Hutzinger et al. 2009). All model pairs for the v-snoRNA are correctly predicted (consistent with C/D box snoRNAs); however, the average z-scores, while negative, were not lower than -1 (Fig. 2a). Thirteen additional base pairs are predicted beyond the annotated $v-$ snoRNA region (orange pairs in Fig. 2b).

ScanFold-Fold was able to detect the terminal hairpin loops of very large hairpin structures found in ncRNAs from two regions. The first hairpin (Fig. S1) occurs in the W repeat region, which generates the stable intronic sequence (sis)RNA-2. This sequence occurs $5-8 \mathrm{X}$ in circulating viral strains and the transcribed sisRNAs accumulate to high abundance in infected cells (Moss \& Steitz 2013) and appear to be important to EBV-induced transformation of human B cells (Bridges et al. 2019; Szymula et al. 2018). The second region is the latent origin of replication (oriP), which is transcribed bidirectionally in lytically reactivated cells. In both oriP transcripts, tandem repeat sequences anneal to each other to form very long hairpins, which interact with human ADAR (adenosine deaminase acting on RNA), are hyper-edited, localized to the nucleus, interact with paraspeckle assembly factors, and play likely roles in immune modulation (Cao et al. 2015a). ScanFold-Scan finds the terminal hairpins of each oriP transcript in windows with less than -5 z-scores, however, the final model reported by the ScanFold-Fold algorithm predicts multiple local inter-repeat interactions (Fig. S2). In both the sisRNA-2 and oriP transcripts, long range base pairs in each hairpin cannot be predicted, as they span distances greater than the window size used (150 nt here). This highlights an important limitation of ScanFold and, indeed, all scanning window approaches.

ScanFold-Fold was able to successfully predict most of the known structure of the EBV encoded small RNA (EBER)1 (Fig. 3), a hyper-abundant viral ncRNA with implications to oncogenesis (Iwakiri 2016; Moss et al. 2014). Similar to the long hairpins discussed above, EBER1 long-range base pairs were not predicted, however, $61 \%$ of the pairs not spanning more than 150 nt were correctly predicted; the majority (14/15) with average z-scores < -2. In EBER2, however, no model base pairs were predicted (Fig. 4) and, indeed, few base pairs with low zscores were predicted. The 21 base pairs with average z-scores $<-1$ do not correspond to any known EBER2 structures. This is due to the poorer predicted metrics for windows spanning the annotated EBER2 region; indeed, windows spanning EBER2 had positive z-scores, indicating a sequence that is potentially ordered to be loosely structured (Andrews et al. 2019). Interestingly, the regions of EBER2 that fall within positive z-score regions correspond to an interaction site that binds to nascent transcripts from the terminal repeat (TR) region of the EBV genome. This interaction facilitates the recruitment of the human PAX5 regulatory protein to the TR genomic region to promote lytic replication (Lee et al. 2015). Interestingly, ScanFold predictions in the TR region find that the EBER2 interaction sites are also spanned by positive z-score windows and $S$ canFold-Fold predictions suggest that these regions are ordered to be unstructured/accessible. 
351

352

353

354

355

356

357

358

359

360

361

362

363

364

365

366

367

368

369

370

371

372

373

374

375

376

377

378

379

380

381

382

383

384

385

386

387

388

389

390

391

392

393

394

395

396

397

398

399

400

\section{New structural motifs in EBV}

In addition to finding known EBV structures, ScanFold predicts many additional motifs with putative functions. We focused our attention on one region which was particularly rich in base pairs predicted to have average z-scores that were exceptionally low $(<-2)$. This region approximately spans EBV bp 48,800 to 50,200 and overlaps several important viral genes (Fig. 5 ). The first cluster of predicted motifs overlaps three lytic genes BFRF1, BFRF2, and BFRF3, which play essential roles in nuclear egress(Farina et al. 2005) (the first step in virion release into infected cells), regulation of late viral gene expression(Aubry et al. 2014), and assist in the assembly of infections particles(Henson et al. 2009), respectively. Each gene overlaps each other, where BFRF2 and BFRF3 both overlap the 3' UTR of BFRF1 and BFRF3 overlaps the 3' UTR of BFRF2. Thus, functional structural motifs can be playing multiple roles in the posttranscriptional control of BFRF1-3. We focused our attention, however, on potential roles in the 3' UTR of BFRF1. A 606 nt fragment sequence (bp 48,856 - 49,461), corresponding to the motifs predicted with average z-scores less than -1 in the BFRF1 3' UTR were further analyzed.

All base pairs predicted by ScanFold-Fold to have average $z$-scores $<-1$ were constrained to be paired to each other and the remaining sequence was refolded using the RNA fold algorithm (the same algorithm used in ScanFold-Scan to predict windows(Lorenz et al. 2011)), which predicts the global MFE structure. This fills in base pairs that are "missing" in the ScanFoldFold consensus (Fig. 6a), allows for potential long-range interactions to occur and allows for base pairs that do not contribute to the low z-score of this region to form. The resulting global model of this region is shown in Fig. 6b. The overall fold can be divided into eight motifs. In most cases a motif was simply the ScanFold-Fold predicted structure. In Motif (M)2 and M6, several base pairs were added to extend helices (Fig. 6b). M4, on the other hand, was predicted to form a multibranch loop structure, which encompasses three ScanFold-Fold predicted hairpins: M4.1, M4.3 and M4.5. To test the resulting model, a phylogenetic analysis was undertaken. The EBV-1 sequence was queried against the nt database using BLASTn (Altschul et al. 1990), which identified 100 putative homologs, all from other EBV strains. Model base pairs were compared to an alignment of EBV-1 and these 100 homologous sequences (Data S1). Canonical base pairing was conserved $99.1 \%$ and, when mutations occurred, they generally preserved structure. Of the 202 base pairs predicted in this region, 35 showed evidence of consistent mutation-a single point mutation which preserves base pairing (Fig. 6b). Only two base pairs showed evidence of compensatory mutation-a double point mutation which preserves base pairing. One putative compensatory mutation occurs in the basal stem of M4.5 and the other in M7 (Fig. 6b).

A second cluster of predicted motifs overlaps the F/Q exons of EBNA1 (Fig. 5). EBNA1 is a key viral gene involved in the replication and partitioning of the EBV genome (Frappier 2012). During latency program I, EBNA1 is the sole expressed protein and transcription begins at the $\underline{Q}$ promoter (Qp; Fig. 7a). This results in the production of a short $Q$ exon, which is spliced upstream of the $U$ leader exon in the EBNA1 5' UTR, which was previously found to contain a structured internal ribosomal entry site (IRES) that stimulates non-canonical (cap-independent) translation (Isaksson et al. 2003b). During lytic reactivation transcription from the $\mathrm{F}$ promoter (Fp) leads to the incorporation of the longer F exon into the $5^{\prime}$ UTR of EBNA1 (includes the $Q$ exon sequence; Fig. 7a). To see if the $\mathrm{ScanFold-Fold} \mathrm{predicted} \mathrm{structural} \mathrm{motifs} \mathrm{would}$ persist in the context of this longer 5' UTR, the entire sequence (plus the start codon), were analyzed using the ScanFold pipeline. Three hairpins were predicted (HP1-3; Fig. 7b) which recapitulate those predicted in the EBV genome scan (Fig. 5). The most significant base pairs

Peer) reviewing PDF | (2020:04:47551:1:1:NEW 5 Aug 2020) 
401 (average z-scores < -2) all occur within HP1, which begins at the first nucleotide transcribed

402

403

404

405

406

407

408

409

410

411

412

413

414

415

416

417

418

419

420

421

422

423

424

425

426

427

428

429

430

431

432

433

434

435

436

437

438

439

440

441

442

443

444

445

446

447

448

449

450

451 from $\mathrm{Fp}$.

In the context of the 5' UTR, the downstream F/Q exon nt are predicted to form a novel hairpin, HP4. No significant base pairing was predicted between the F/Q and U leader exons. Interestingly, no base pairs were predicted in the IRES-containing $U$ leader exon with average $z$-scores $<-1$. The negative $z$-score $(<0$ but $>-1)$ base pairs predicted here do not correspond to any found in the EBNA1 IRES model structure (Moss et al. 2014). After constraining ScanFold-Fold predicted base pairs $(<-1)$ and re-folding the 5' UTR, however, four additional hairpins (HP5-8) were predicted (Fig. 7c). HP7 and HP8 are found in the current consensus model of the EBNA1 IRES (Rfam ID\# RF00448) in the Rfam database (Burge et al. 2013; Daub et al. 2015; Gardner et al. 2009; Griffiths-Jones et al. 2003; Griffiths-Jones et al. 2005; Kalvari et al. 2018a; Kalvari et al. 2018b; Nawrocki et al. 2015). HP6 is a novel predicted configuration of the IRES nucleotides and HP5 is a novel predicted hairpin upstream of the IRES, which partially overlaps (incorporating two nucleotides of) the F/Q exon (Fig. 7c). HP6-8 are predicted to occur within a multibranch loop structure that sits above an extensive basal stem structure.

To check for potential conservation of the model structure, as well as identify putative consistent and compensatory changes to support model base pairs, a phylogenetic comparison was performed using an alignment of $49 \mathrm{EBV}$ sequences (Data S2). Overall conservation of canonical base pairing was $98.0 \%$. There were 22 putative consistent mutations and 3 compensatory mutations identified (Fig. 7c). These putative compensatory mutations are found in HP4, HP6 and HP7. No consistent or compensatory mutations were identified within the long basal stem under HP6-8.

\section{Experimental analysis of a novel structured region}

To test potential functions of the motifs overlapping BFRF1, BFRF2, and BFRF3, four constructs were generated that comprise the entire WT structural region and three fragments (containing M1-2, M3-6, and M7-8; Fig. 6c), which were placed downstream (within the 3' UTR) of Renilla Luciferase $(R L)$ and expressed in human cell lines. Two values were measured for each construct, as well as an empty vector control (Fig. 6d): the Luciferase activity fold-change (Luciferase activity is a measure of protein expression levels) and the translational efficiency (which normalizes protein levels using the RNA abundance measured by RT-qPCR); see Methods. Compared to the control (C), the WT construct had reduced Luciferase activity, but a $\sim 7$-fold increase in translational efficiency. The M1-2 construct saw marked increases in Luciferase activity ( $\sim$-fold) and translational efficiency ( $\sim 36$-fold increase). M3-6 reduced the Luciferase activity to wild type levels, however translational efficiency was roughly cut in half compared to control. M7-8 reduced the Luciferase activity and translational efficiency below WT levels (by roughly $60 \%$ in both cases). These results indicate the presence of repressive elements in the region spanned by M3-8 and stimulatory elements in M1-2.

\section{Structural motifs beyond EBV}

Beyond EBV, RNA secondary structures have been previously discovered in KSHV. A recent analysis of the KSHV PAN ncRNA (Sztuba-Solinska et al. 2017) (an RNA transcript that is important to late lytic gene expression and the release of progeny virions (Borah et al. 2011; Sun et al. 1996)) generated a global secondary structure model for PAN based on comprehensive SHAPE probing under in/ex cellulo, and in/ex virio in vitro conditions. ScanFold-Fold results for the KSHV genomic region encoding PAN recapitulated many features of the experimentally-informed global model (Fig. 8). Notable, however, is the lack of

Peer] reviewing PDF | (2020:04:47551:1:1:NEW 5 Aug 2020) 
452

453

454

455

456

457

458

459

460

461

462

463

464

465

466

467

468

469

470

471

472

473

474

475

476

477

478

479

480

481

482

483

484

485

486

487

488

489

490

491

492

493

494

495

496

497

498

499

500

501

highly negative average z-score base pairs from the ScanFold-Fold predictions: indeed, the majority of base pairs were predicted with negative average $z$-scores that did not fall below -1 . Base pairs predicted with average $z$-scores below -1 clustered between PAN nucleotides 137 to 327 ; the only motif from the previous global model with average z-score $<-1$ fell within this region (H8; Fig. 8).

Motif base pairs (after refolding; see Methods) are 99.4\% conserved in an alignment of PAN homologs (Data S3) and the little variation observed was consistent with base pairing. 11 out of 19 motifs predicted by ScanFold appeared in the global model (Fig. 8) (Sztuba-Solinska et al. 2017) and, with small variations (e.g. see results of the PAN ENE below), recapitulated the experimentally-guided model base pairs. The nucleotides in the $8 \mathrm{ScanF}$ ld motifs that were not found in the global model were, in that model, generally found in longer range interactions and/or showed high SHAPE reactivity under various probing conditions (indicating singlestranded or loose structure). These results are consistent with the observation that regions with z-scores greater than -1 generally corresponded to higher SHAPE reactivities (Andrews \& Moss $2019 b$ ) (potentially due to the lack of a predominant secondary structure and/or the presence of primary sequence motifs whose activity is facilitated by being unstructured). For example, the motif predicted for nucleotides 34 to 91 contains the Mta responsive element (MRE; a protein binding site that stabilizes PAN (Massimelli et al. 2011; Tunnicliffe et al. 2019) partially occluded within a weak stem (comprised of GU and AU base pairs; Fig. 8). In the global model the MRE site is highly reactive to SHAPE probing (under ex virio conditions) and the remaining motif bases are contained within long-range helices ( $\mathrm{H} 2$ and $\mathrm{H} 3$ in Figure 1 of (Sztuba-Solinska et al. 2017)). The motif spanning nucleotides 343 to 376 , however, is modeled as a small alternative hairpin (H12 in Figure 1 of (Sztuba-Solinska et al. 2017)) in a looped out region with moderate SHAPE reactivity (under ex virio conditions).

The best characterized (in structure and function) motif is the ENE (expression and nuclear retention element) found in the $3^{\prime}$ end of the KSHV PAN ncRNA (Conrad et al. 2006). This element was found to increase the stability/lifetime of intronless transcripts, such as the PAN RNA, via the sequestration of the PAN poly $(A)$ tail in an extensive triple-helix structure with bulged uracil nucleotides in the ENE (Mitton-Fry et al. 2010). In the analysis of the KSHV genome, ScanFold-Fold was able deduce the location of the ENE and predict a secondary structure in agreement (Fig. 8) with the crystal structure of the PAN ENE(Mitton-Fry et al. 2010) (crystalized nucleotides highlighted in green) and the SHAPE directed model (of the cytoplasmic and ex virio RNA; Figures S4 and S7 of (Sztuba-Solinska et al. 2017)).

Beyond the KSHV PAN there are many base pairs predicted with higher significance: there are 39906 base pairs that have average z-scores $<-1$ and $9446<-2$ predicted in the KSHV genome. The values for KSHV are on par with predictions for other herpesviruses (Table 1), indicating significant fractions of each genome likely encode functional RNA secondary structures (all results available on the RNAstructuromeDB (Andrews et al. 2017) and in Supplemental Dataset (https://zenodo.org/record/3964325).

\section{Discussion}

Innovations present in the ScanFold approach made this current report possible. The initial ScanFold-Scan analysis generates metrics of local RNA folding that allow for genome-wide comparisons between human herpesviruses. A key finding of this study is that EBV (types 1 and 2) both have evidence of containing more sequences that are ordered to form (likely functional) 
502 RNA structures than any other herpesviruses: e.g. the average z-score for EBV is $\sim 2 X$ that of 503 the next lowest (KSHV; Table 1). It should be noted, however, that even for the genome with the least negative average z-score, HHV-6B (-0.09; Table 1), significant percentages of the genome are spanned by low z-score windows. These give rise to many predicted base pairs with likely functionality: e.g. for HHV-6B $5.8 \%$ and $0.8 \%$ of the 607,730 base pairs predicted by ScanFold-Fold have average z-scores $<-1$ (28,525 bp) and < -2 (4,780 bp), respectively. Thus, although RNA secondary structure is predicted to play greater roles in EBV, all human herpesviruses are likely to utilize regulatory RNA structure to a larger extent than previously appreciated. The results of these analyses are made available on the RNAstructuromeDB (Andrews et al. 2017) to facilitate their usage by a wide array of researchers interested in human herpesviruses. Here, results can be browsed alongside genomic annotations (which have been loaded from NCBI for each genome) or any JBrowse compatible file (Buels et al. 2016) a user has available. As an example, for EBV-1 we include additional tracks alongside ScanFold results to help identify regions of interest; the Mclntosh lab has generated RNA sequencing data for the EBV-1 genome as it transitions from a latent to a lytically active state (Frey et al. 2020). The sequencing data from this study can now be seen as a coverage tracks and allows users to quickly assess whether regions highlighted by ScanFold fall within actively transcribed regions.

In addition to facilitating global comparisons, the ScanFold approach defines specific motifs of likely function. All predicted motifs containing at least one base pair with an average z-score < 2 have been modeled, annotated, and compiled into individual PDF files for easy viewing (Supplemental Dataset - https://zenodo.org/record/3964325). In addition to presenting the thermodynamic properties for each motif, the NCBI gene features overlapping the motif have been listed. In EBV-1, ScanFold was able to identify the locations of and recapitulate elements of known functional structured RNAs (Figs. 1-3 and Figs. S1-S2). For example, although ScanFold is not explicitly a miRNA discovery program, it does a strikingly good job of deducing pre-miRNA hairpins. All known EBV miRNAs are contained within hairpins formed by base pairs with z-averages < - 1 and the majority have base pairs $<-2$ (RNAstructuromeDB and Supplemental Dataset - https://zenodo.org/record/3964325): e.g. the BART miRNA cluster shown in Fig. 1. The exceptionally low average z-scores of pre-miRNA base pairs indicates a particular bias in their sequence order, highlighting the strict structural requirements of miRNA maturation (Lee et al. 2002; Zeng \& Cullen 2003).

ScanFold results may also provide information on the processing of another EBV ncRNA, vsnoRNA1. In addition to predicting a C/D box snoRNA-like hairpin in the annotated v-snoRNA1 region, additional base pairs are predicted that lengthen the hairpin (Fig. 2). Interestingly, these additional predicted base pairs have lower average z-scores than those of the core v-snoRNA1 structure. The extended hairpin predicted here may be a precursor structure, required to mature the v-snoRNA1 (Hutzinger et al. 2009; Matera et al. 2007). It is also interesting that, while being negative, the majority of core base pairs are not predicted to have average z-scores below -1 . This may be to facilitate intermolecular RNA-RNA interactions with target sites on the host RNAs: e.g. utilizing the D' and D sites(Hutzinger et al. 2009) annotated on Fig. 2.

Similar results were found in the EBER2 and TR regions of EBV, which form biologically essential intermolecular RNA-RNA interactions with each other (Fig. 4) (Lee et al. 2015). But for several isolated base pairs, the EBER2 TR interaction region is predicted to have unusually stable base pairs (Fig. 4a) as are the two interaction sites in the TR RNA (Fig. 4b), which occur within a region of highly positive predicted z-score-indicating they may be ordered to be unusually unstable (presumably to facilitate intermolecular base pairing). None of the EBER2 
552 reference structure (Rfam ID\# RF02712) base pairs are predicted by ScanFold-Fold (Fig.

553 4c); emphasizing its loose secondary structure, which is also supported by previous

554 experimental analyses (Lee et al. 2015).

In contrast to EBER2, EBER1 is predicted to contain base pairs with average z-scores < -2, indicating a high degree of ordered structure, which is corroborated by experimental analyses(Glickman et al. 1988; Lee et al. 2015). These low z-score base pairs form three hairpins in the reference structure (Rfam ID\# RF01789), with the two remaining reference structure hairpins being predicted by base pairs with average z-score $<-1$ (Fig. 3 ). The remaining $46 \%$ of base pairs in the reference structure that were not predicted by ScanFoldFold highlight an important limitation of ScanFold: it cannot predict longer range base pairs (due to the limitation of the window size used). To predict the reference structure, however, ScanFold-Fold results can aid modeling by providing base pairs to use as constraints (e.g. Fig. 3c). A priori knowledge of the final transcript sequence is, however, essential here. This highlights another important limitation of whole genome scans: defining the boundaries of a structured domain or ncRNA. In EBER1, for example, 10 base pairs with average z-scores $<-2$ are predicted between EBER1 and upstream EBV sequences; as is a single base pair < -1 predicted to occur downstream (Fig. 3a). The EBER1 sequence can be co-transcribed as part of intronic sequences for latent membrane protein (LMP)2 and these structures between EBER2 and flanking sequence could play some role in splicing; however, additional work is needed to test this speculative hypothesis.

Beyond previously described structures in EBV, ScanFold uncovered many novel motifs; we focused on structures predicted in a particular "hot spot" with base pairs having average zscores < -2 (indicating highly ordered sequences/structures; Fig. 5). The motifs overlapping BFRF1-3 do so primarily in 3' UTR sequences (exclusively in BFRF1 and partially in BFRF2), thus their hypothesized roles in regulation of gene expression; as 3' UTRs are particularly rich areas for post-transcriptional control elements (Mayr 2017). Indeed, we found that inclusion of motifs $\mathrm{M} 1$ and $\mathrm{M} 2$ into a reporter construct stimulated expression of luciferase, while motifs M3 to M6 and M7 to M8 both suppressed expression (Fig. 6b). Many factors can be playing roles in the observed effects of these motifs. For example, multiple host regulatory proteins are predicted to target M1-8 (Data S5) as are both host and viral miRNAs (Table S1). Motif structure can play many roles here: by forming specific recognizable motifs; occluding or presenting primary sequence motifs, or by altering the relative positioning of regulatory motifs. Much additional work is required to validate predicted interactions in this region and to define the exact roles of the conserved and unusually stable secondary structures discovered here: e.g. introducing minimal mutations to modulate predicted RNA secondary structures can help determine to what extent RNA structure plays a role in the observed results.

Similarly, we identified four highly stable/conserved hairpins in the F/Q exon of EBNA1, which are, essentially, appended as a single domain to the 5' UTR of this critical viral gene during lytic replication. An interesting observation was that these novel elements had base pairs that were predicted with average z-scores that were much lower than those of the known structures in the downstream $U$ leader exon (Isaksson et al. 2003a) (Fig. 7). Indeed, the EBNA1 IRES base pairs could only be predicted after refolding the 5' UTR with upstream sequences constrained based on the ScanFold-Fold predicted base pairs. Phylogenic support is found for all model structures, indicating that all have likely importance; however, the IRES shows much less evidence of unusual stability. Perhaps, as in the previously discussed examples, some flexibility is required in the IRES for its function. What potential roles then, can be proposed for the rigidly 
602 defined structures in the F/Q exon? One interesting possibility is that structure here is inhibiting 603 cap-dependent translation (e.g. by sequestering the 5' end of the mRNA in the exceptionally 604 stable HP1; Fig 7), potentially driving the use of the EBNA1 IRES in non-canonical translation. 605 This hypothesis is supported by data from a previous study (Isaksson et al. 2003b), which 606 showed that inclusion of this structured upstream sequence reduced relative luciferase activities of all tested constructs. Furthermore, when the IRES domain was not present, constructs containing the upstream structures had the lowest relative luciferase observed levels.

\section{Conclusion}

A significant outcome of this study was the prediction of extensive functional RNA structures throughout other human herpesviruses. It is likely that, similar to EBV, these motifs are playing important roles in the regulation of herpesvirus biology, infection and disease. Additional studies of these motifs will provide many insights, which are facilitated by making all results available on the RNAstructuromeDB. Similarly, a web server is available (Andrews et al. 2019; Andrews \& Moss 2019b) for running ScanFold calculations and for aiding in mutational design of constructs used in experimental assays (e.g. using RNA2 DMut(Moss 2018b)).

\section{Acknowledgements}

This research was supported by NIH/NIGMS grants R00GM112877 and R01GM133810, as well as by startup funds from the Roy J. Carver Charitable Trust. Thanks also to Nuwanthika Kumarasinghe for her assistance.

\section{Competing Interests}

The authors declare no competing interests.

\section{Figure Captions}

Figure 1. Results for the EBV-BART miRNA cluster. (a) IGV visualization of results. EBV-1 genome coordinates are followed by $\mathrm{ScanFold-Fold} \mathrm{predicted} \mathrm{base} \mathrm{pairs} \mathrm{represented} \mathrm{as} \mathrm{arcs}$ (colored blue and green for bp with average z-score < -2 and -1 , respectively, and yellow for bp with negative average $z$-scores $>-1$ ), and the genome sequence $(A, C, G$, and $T$ are in green, blue, orange and red, respectively). Below this is a bar-graph showing the window z-score values predicted by ScanFold-Scan (each bar sits at the $1^{\text {st }}$ nt of the window and is colored red or blue for negative/positive values, respectively; the range of values are indicated in brackets). A cartoon of the gene structure of EBV-1 is shown in blue with the location of BART miRNAs annotated. (b) At the bottom is the ScanFold-Fold secondary structure model for the EBV-BART3 pre-miRNA hairpin. Mature sequences are annotated in blue and red.

641 Figure 2. Results for the v-snoRNA1. (a) IGV visualization of results. EBV-1 genome coordinates are followed by ScanFold-Fold predicted base pairs represented as arcs (colored green for bp with average $z$-score $<-1$ and yellow for bp with negative average $z$-scores $>-1$ ), the genome sequence (A, C, G, and T are in green, blue, orange and red, respectively). (b) To the right is the ScanFold-Fold secondary structure model for v-snoRNA1 where additional sequences, beyond the annotated v-snoRNA1 sequence is colored orange. Proposed D' and D interaction sites with host rRNA sequences are indicated in pink and blue, respectively. 
648 Figure 3. Results for EBER1. (a) IGV visualization of results. At the top of IGV, EBV-1 genome 649 coordinates are shown, followed by ScanFold-Fold predicted base pairs represented as arcs 650 and the location of EBER1 is indicated by the blue cartoon. Base pairs in the reference EBER1 651 structure correctly predicted by ScanFold-Fold are highlighted in green on the secondary 652 structure in (b). (c) indicates the correctly predicted pairs after refolding the EBER1 sequence 653 using ScanFold-Fold bp with average z-scores $<-2$ as constraints.

654 Figure 4. Results for EBER2 and its interaction sites on the terminal repeat RNA sequence. (a) 655 EBV-1 genome coordinates are followed by ScanFold-Fold predicted base pairs represented 656 as arcs (colored blue and green for bp with average $z$-score $<-2$ and -1 , respectively, and 657 yellow for bp with negative average z-scores $>-1$ ), the genome sequence $(A, C, G$, and $T$ are in green, blue, orange and red, respectively). The EBER2 encoding region is indicated with a blue cartoon, this includes an annotation of the terminal repeat (TR) interacting nucleotides in EBER2. (b) ScanFold-Fold predicted base pairs for the TR region, the corresponding binding region to EBER2, represented in panel $A$, with the addition of annotations for the two EBER2 interaction regions (sites $A$ and $B$ ) and a bar-graph showing the window z-score values predicted by ScanFold-Scan (each bar sits at the $1^{\text {st }} \mathrm{nt}$ of the window and is colored red or blue for negative/positive values, respectively; the range of values are indicated in brackets). (c) Secondary structure model of EBER2 with the TR interacting nucleotides annotated in orange and red for Site A and B, respectively. Model duplexes for each interaction are shown to the 667 right.

668 Figure 5. Results for the EBV-1 genome region partially encoding BFRF1-3 and part EBNA1. 669 EBV-1 genome coordinates are followed by ScanFold-Fold predicted base pairs represented as colored arcs and the location of each gene is indicated by the blue cartoon. The light blue highlighted region is the region used for subsequent experimental analysis.

672 Figure 6. Results for the region partially overlapping BFRF1-3. (a) IGV visualization of results.

673 At the top of IGV, EBV-1 genome coordinates are shown, followed by ScanFold-Fold predicted base pairs represented as colored arcs. (b) Secondary structure models predicted after energy minimization using ScanFold-Fold base pairs (average z-score < - 1 ) as constraints. Eight motifs (M1-8) were defined (labeled in both the (a) and (b)). Nucleotides with mutations preserving the shown base pair are circled on the structure models. (c) Cartoon showing the locations of three regions (colored green, purple, and red) that were added to downstream of Renilla luciferase (RL; diagrams of constructs are shown with RL in yellow and the added fragments of EBV-1 in colors corresponding to the cartoon). (d) Results of luciferase assays for each construct; the translational efficiency and luciferase mRNA and protein foldchange (vs. empty vector control [C]) are plotted for C, wild type (WT) and three fragments of the structured region. The translational efficiencies reported here were calculated by dividing the relative protein abundance of $R L$ (RRR values) by mRNA levels of $R L$ (2- $2^{-\Delta \Delta C T}$ values); see methods for detail and Table S2 for raw values.

Figure 7. Results for the EBNA1 5' UTR. (a) Cartoon showing the EBV-1 genome region with the Fp (used in lytic replication) and $\mathrm{Qp}$ (used in latency type I and II) promoters annotated alongside the $F / Q, U$, and $K$ exons (with start codon colored green).(b) ScanFold-Fold 689 predicted base pairs represented as colored arcs above the sequence of the EBNA1 5' UTR 690 formed after transcription from Fp. Spliced exon sequences are highlighted and labeled below 691 the arc diagram structure (with highlights colored as in the cartoon). (c) Secondary structure 
692

693

694

695

696

697

698

699

700

701

702

703

704

705

706

707

708

709

710

711

712

713

714

715

716

717

718

719

720

721

722

723

724

725

726

727

728

729

730

731

model of the EBNA1 5' UTR based on energy minimization using ScanFold-Fold base pairs with average z-scores < -1 as constraints. The locations of exon starts and the start codon are annotated; as well, nt that show evidence of undergoing structure-preserving mutations are circled.

Figure 8. Results for KSHV PAN. (a) IGV visualization of results with KSHV genome coordinates followed by $\mathrm{ScanFold-Fold}$ predicted base pairs represented as arcs (colored green for bp with average $z$-score $<-1$, yellow for bp with negative average $z$-scores $>-1$, and grey for $>0$ ), the genome sequence (A, C, G, and T are in green, blue, orange and red, respectively). (b) The bottom panel contains motif secondary structures predicted by ScanFold-Fold depicted using VARNA (Darty et al. 2009). The MRE is represented as an orange highlight on the arc diagrams and as orange bps in the secondary structures. The nucleotides from the ENE motif which have been crystalized(Mitton-Fry et al. 2010) are highlighted in green.

\section{Supplemental Information}

Supplemental Dataset. ScanFold output for all genomes. Raw output alongside files used to visualize data on IGV or other genome viewers. Please see README for descriptions of each file. Additional information on using $\mathrm{ScanFold}$ and analyzing output files is available in (Andrews et al. 2019). Dataset available as a Zenodo repository at: https://zenodo.org/record/3964325 or from the RNAStructuromeDB at https://structurome.bb.iastate.edu/herpesvirus.

Supplemental Data S1. MAFFT alignment of the BFRF1 3'UTR using 100 BLASTn deduced homologous sequences. Closely related sequence alignments can show conservation at both the primary sequence level and secondary structure level. Mutations which conserve base pairing of the secondary structure (i.e. point and compensatory mutations) are evidence of potentially functional structures as evolution is favoring the conservation of the structure across species. Significant ScanFold predicted structures can be queried for primary and secondary conservation to further home in on functional regions. The EBV BFRF1 3' UTR is the first sequence in the file, followed by the homologous sequences.

Supplemental Data S2. MAFFT alignment of the EBNA1 3' UTR using 48 BLASTn deduced homologous sequences. The purpose of MAFFT alignments and importance of resulting data is described above (Supplemental Data S1). The EBNA1 3' UTR is the first sequence in the file, followed by the homologous sequences.

Supplemental Data S3. MAFFT alignment of the PAN RNA using 33 BLASTn deduced homologous sequences. The purpose of MAFFT alignments and importance of resulting data is described above (Supplemental Data S1). The KSHV PAN RNA is the first sequence in the file, followed by the homologous sequences.

Supplemental Data S4. RBPMap predictions for BFRF1 3' UTR. File contains predicted binding site location, motif sequence, k-mer sequence, $p$-value, and z-score for each prediction. RBP 
732 binding regions can be cross referenced to ScanFold predicted structures and to miRNA sites,

733 which facilitates hypothesis generation regarding the function of a motif and/or region.

734 Supplemental Table S1. A list of miRDuplex predictions for BFRF1 3' UTR. Predicted

735 miRNAs are listed along with sequence and positional information of the corresponding target

736 (BFRF1 3' UTR). miRNA binding regions can be cross referenced to ScanFold predicted

737 structures and to RBP sites, which facilitates hypothesis generation regarding the function of a

738 motif and/or region.

739 Supplemental Table S2. Results of dual luciferase assays and RT-qPCR for motifs in the

740 overlapping BFRF1-3 region. These values were used to generate the bar/line graph in Fig. $6 \mathrm{~d}$.

741 Supplemental Figure S1. ScanFold-Fold results for the ebv-sisRNA-2 Terminal Hairpin.

742 Supplemental Figure S2. ScanFold results for the oriP Region of the EBV genome.

743

744

745

746

747

748

749

750

751

752

753

754

755

756

757

758

759

760

761

762

763

764

765

766

767

768

769

770

771

772

773

774

775

776

\section{References}

Altschul SF, Gish W, Miller W, Myers EW, and Lipman DJ. 1990. Basic local alignment search tool. J Mol Biol 215:403-410. 10.1016/S0022-2836(05)80360-2

Andrews RJ, Baber L, and Moss WN. 2017. RNAStructuromeDB: A genome-wide database for RNA structural inference. Sci Rep 7:17269. 10.1038/s41598-017-17510-y

Andrews RJ, Baber L, and Moss WN. 2019. Mapping the RNA structural landscape of viral genomes. Methods. 10.1016/j.ymeth.2019.11.001

Andrews RJ, and Moss WN. 2019a. Computational approaches for the discovery of splicing regulatory RNA structures. Biochim Biophys Acta Gene Regul Mech. 10.1016/j.bbagrm.2019.04.007

Andrews RJ, and Moss WN. 2019b. Mapping the RNA structural landscape of viral genomes. Methods. 10.1016/j.ymeth.2019.11.001

Andrews RJ, Roche J, and Moss WN. 2018. ScanFold: an approach for genome-wide discovery of local RNA structural elements-applications to Zika virus and HIV. PeerJ 6:e6136. 10.7717/peerj.6136

Aubry V, Mure F, Mariame B, Deschamps T, Wyrwicz LS, Manet E, and Gruffat H. 2014. Epstein-Barr virus late gene transcription depends on the assembly of a virus-specific preinitiation complex. $J$ Virol 88:12825-12838. 10.1128/JVI.02139-14

Bevilacqua PC, Ritchey LE, Su Z, and Assmann SM. 2016. Genome-Wide Analysis of RNA Secondary Structure. Annu Rev Genet 50:235-266. 10.1146/annurev-genet-120215-035034

Borah S, Darricarrere N, Darnell A, Myoung J, and Steitz JA. 2011. A viral nuclear noncoding RNA binds re-localized poly $(\mathrm{A})$ binding protein and is required for late KSHV gene expression. PLoS Pathog 7:e1002300. 10.1371/journal.ppat.1002300

Bridges R, Correia S, Wegner F, Venturini C, Palser A, White RE, Kellam P, Breuer J, and Farrell PJ. 2019. Essential role of inverted repeat in Epstein-Barr virus IR-1 in B cell transformation; geographical variation of the viral genome. Philos Trans R Soc Lond B Biol Sci 374:20180299. 10.1098/rstb.2018.0299

Buels R, Yao E, Diesh CM, Hayes RD, Munoz-Torres M, Helt G, Goodstein DM, Elsik CG, Lewis SE, Stein L, and Holmes IH. 2016. JBrowse: a dynamic web platform for genome visualization and analysis. Genome Biol 17:66. 10.1186/s13059-016-0924-1

Burge SW, Daub J, Eberhardt R, Tate J, Barquist L, Nawrocki EP, Eddy SR, Gardner PP, and Bateman A. 2013. Rfam 11.0: 10 years of RNA families. Nucleic Acids Res 41:D226-232. 10.1093/nar/gks1005

Cao S, Moss W, O'Grady T, Concha M, Strong MJ, Wang X, Yu Y, Baddoo M, Zhang K, Fewell C, Lin Z, Dong Y, and Flemington EK. 2015a. New Noncoding Lytic Transcripts Derived from the Epstein-

Peer] reviewing PDF | (2020:04:47551:1:1:NEW 5 Aug 2020) 
783

784

785

786

787

788

789

790

791

792

793

794

795

796

797

798

799

800

801

802

803

804

805

806

807

808

809

810

811

812

813

814

815

816

817

818

819

820

821

822

823

824

Barr Virus Latency Origin of Replication, oriP, Are Hyperedited, Bind the Paraspeckle Protein, NONO/p54nrb, and Support Viral Lytic Transcription. J Virol 89:7120-7132. 10.1128/JVI.0060815

Cao S, Strong MJ, Wang X, Moss WN, Concha M, Lin Z, O'Grady T, Baddoo M, Fewell C, Renne R, and Flemington EK. 2015b. High-throughput RNA sequencing-based virome analysis of 50 lymphoma cell lines from the Cancer Cell Line Encyclopedia project. J Virol 89:713-729. 10.1128/JVI.0257014

Clote P, Ferre F, Kranakis E, and Krizanc D. 2005. Structural RNA has lower folding energy than random RNA of the same dinucleotide frequency. RNA 11:578-591. 10.1261/rna.7220505

Conrad NK, Mili S, Marshall EL, Shu MD, and Steitz JA. 2006. Identification of a rapid mammalian deadenylation-dependent decay pathway and its inhibition by a viral RNA element. Mol Cell 24:943-953. 10.1016/j.molcel.2006.10.029

Darty K, Denise A, and Ponty Y. 2009. VARNA: Interactive drawing and editing of the RNA secondary structure. Bioinformatics 25:1974-1975. 10.1093/bioinformatics/btp250

Daub J, Eberhardt RY, Tate JG, and Burge SW. 2015. Rfam: annotating families of non-coding RNA sequences. Methods Mol Biol 1269:349-363. 10.1007/978-1-4939-2291-8_22

Draborg AH, Duus K, and Houen G. 2013. Epstein-Barr virus in systemic autoimmune diseases. Clin Dev Immunol 2013:535738. 10.1155/2013/535738

Elgui de Oliveira D, Muller-Coan BG, and Pagano JS. 2016. Viral Carcinogenesis Beyond Malignant Transformation: EBV in the Progression of Human Cancers. Trends Microbiol 24:649-664. 10.1016/j.tim.2016.03.008

Farina A, Feederle R, Raffa S, Gonnella R, Santarelli R, Frati L, Angeloni A, Torrisi MR, Faggioni A, and Delecluse HJ. 2005. BFRF1 of Epstein-Barr virus is essential for efficient primary viral envelopment and egress. J Virol 79:3703-3712. 10.1128/JVI.79.6.3703-3712.2005

Frappier L. 2012. The Epstein-Barr Virus EBNA1 Protein. Scientifica (Cairo) 2012:438204. 10.6064/2012/438204

Frey TR, Brathwaite J, Li X, Burgula S, Akinyemi IA, Agarwal S, Burton EM, Ljungman M, Mclntosh MT, and Bhaduri-McIntosh S. 2020. Nascent Transcriptomics Reveal Cellular Prolytic Factors Upregulated Upstream of the Latent-to-Lytic Switch Protein of Epstein-Barr Virus. J Virol 94. 10.1128/JVI.01966-19

Gardner PP, Daub J, Tate JG, Nawrocki EP, Kolbe DL, Lindgreen S, Wilkinson AC, Finn RD, Griffiths-Jones S, Eddy SR, and Bateman A. 2009. Rfam: updates to the RNA families database. Nucleic Acids Res 37:D136-140. 10.1093/nar/gkn766

Glickman JN, Howe JG, and Steitz JA. 1988. Structural-Analyses of Eber1 and Eber2 RibonucleoproteinParticles Present in Epstein-Barr Virus-Infected Cells. Journal of Virology 62:902-911.

Griffiths-Jones S, Bateman A, Marshall M, Khanna A, and Eddy SR. 2003. Rfam: an RNA family database. Nucleic Acids Res 31:439-441.

Griffiths-Jones S, Moxon S, Marshall M, Khanna A, Eddy SR, and Bateman A. 2005. Rfam: annotating non-coding RNAs in complete genomes. Nucleic Acids Res 33:D121-124. 10.1093/nar/gki081

Gruber AR, Findeiss S, Washietl S, Hofacker IL, and Stadler PF. 2010. RNAz 2.0: improved noncoding RNA detection. Pac Symp Biocomput:69-79.

Henson BW, Perkins EM, Cothran JE, and Desai P. 2009. Self-assembly of Epstein-Barr virus capsids. J Virol 83:3877-3890. 10.1128/JVI.01733-08

Hutzinger R, Feederle R, Mrazek J, Schiefermeier N, Balwierz PJ, Zavolan M, Polacek N, Delecluse HJ, and Huttenhofer A. 2009. Expression and processing of a small nucleolar RNA from the Epstein-Barr virus genome. PLoS Pathog 5:e1000547. 10.1371/journal.ppat.1000547

Isaksson A, Berggren M, and Ricksten A. 2003a. Epstein-Barr virus U leader exon contains an internal ribosome entry site. Oncogene 22:572-581. 10.1038/sj.onc.1206149

Peer] reviewing PDF | (2020:04:47551:1:1:NEW 5 Aug 2020) 
825

826

827

828

829

830

831

832

833

834

835

836

837

838

839

840

841

842

843

844

845

846

847

848

849

850

851

852

853

854

855

856

857

858

859

860

861

862

863

864

865

866

867

868

869

870

871

872

Isaksson A, Berggren M, and Ricksten A. 2003b. Epstein-Barr virus U leader exon contains an internal ribosome entry site. Oncogene 22:572-581. 10.1038/sj.onc.1206149

Iwakiri D. 2016. Multifunctional non-coding Epstein-Barr virus encoded RNAs (EBERs) contribute to viral pathogenesis. Virus Res 212:30-38. 10.1016/j.virusres.2015.08.007

Kalvari I, Argasinska J, Quinones-Olvera N, Nawrocki EP, Rivas E, Eddy SR, Bateman A, Finn RD, and Petrov Al. 2018a. Rfam 13.0: shifting to a genome-centric resource for non-coding RNA families. Nucleic Acids Res 46:D335-D342. 10.1093/nar/gkx1038

Kalvari I, Nawrocki EP, Argasinska J, Quinones-Olvera N, Finn RD, Bateman A, and Petrov Al. 2018b. NonCoding RNA Analysis Using the Rfam Database. Curr Protoc Bioinformatics 62:e51. 10.1002/cpbi.51

Katoh K, Asimenos G, and Toh H. 2009. Multiple alignment of DNA sequences with MAFFT. Methods Mol Biol 537:39-64. 10.1007/978-1-59745-251-9_3

Katoh K, and Frith MC. 2012. Adding unaligned sequences into an existing alignment using MAFFT and LAST. Bioinformatics 28:3144-3146. 10.1093/bioinformatics/bts578

Katoh K, Kuma K, Miyata T, and Toh H. 2005. Improvement in the accuracy of multiple sequence alignment program MAFFT. Genome Inform 16:22-33.

Katoh K, Misawa K, Kuma K, and Miyata T. 2002. MAFFT: a novel method for rapid multiple sequence alignment based on fast Fourier transform. Nucleic Acids Res 30:3059-3066.

Katoh K, Rozewicki J, and Yamada KD. 2017. MAFFT online service: multiple sequence alignment, interactive sequence choice and visualization. Briefings in Bioinformatics:bbx108-bbx108. $10.1093 / \mathrm{bib} / \mathrm{bbx} 108$

Katoh K, and Standley DM. 2013. MAFFT multiple sequence alignment software version 7: improvements in performance and usability. Mol Biol Evol 30:772-780. 10.1093/molbev/mst010

Katoh K, and Standley DM. 2016. A simple method to control over-alignment in the MAFFT multiple sequence alignment program. Bioinformatics 32:1933-1942. 10.1093/bioinformatics/btw108

Katoh K, and Toh H. 2008a. Improved accuracy of multiple ncRNA alignment by incorporating structural information into a MAFFT-based framework. BMC Bioinformatics 9:212. 10.1186/1471-2105-9212

Katoh K, and Toh H. 2008b. Recent developments in the MAFFT multiple sequence alignment program. Brief Bioinform 9:286-298. 10.1093/bib/bbn013

Katoh K, and Toh H. 2010. Parallelization of the MAFFT multiple sequence alignment program. Bioinformatics 26:1899-1900. 10.1093/bioinformatics/btq224

Kieff E, and Rickinson AJe. 2001. Fields virology.2511-2574.

Kumarasinghe N, and Moss WN. 2019. Analysis of a structured intronic region of the LMP2 pre-mRNA from EBV reveals associations with human regulatory proteins and nuclear actin. BMC Res Notes 12:33. 10.1186/s13104-019-4070-1

Kuraku S, Zmasek CM, Nishimura O, and Katoh K. 2013. aLeaves facilitates on-demand exploration of metazoan gene family trees on MAFFT sequence alignment server with enhanced interactivity. Nucleic Acids Res 41:W22-28. 10.1093/nar/gkt389

Lee N, Moss WN, Yario TA, and Steitz JA. 2015. EBV noncoding RNA binds nascent RNA to drive host PAX5 to viral DNA. Cell 160:607-618. 10.1016/j.cell.2015.01.015

Lee N, Pimienta G, and Steitz JA. 2012. AUF1/hnRNP D is a novel protein partner of the EBER1 noncoding RNA of Epstein-Barr virus. RNA 18:2073-2082. 10.1261/rna.034900.112

Lee N, Yario TA, Gao JS, and Steitz JA. 2016. EBV noncoding RNA EBER2 interacts with host RNA-binding proteins to regulate viral gene expression. Proc Natl Acad Sci U S A 113:3221-3226. 10.1073/pnas.1601773113

Lee $\mathrm{Y}$, Jeon K, Lee JT, Kim S, and Kim VN. 2002. MicroRNA maturation: stepwise processing and subcellular localization. EMBO J 21:4663-4670. 10.1093/emboj/cdf476

Peer) reviewing PDF | (2020:04:47551:1:1:NEW 5 Aug 2020) 
873

874

875

876

877

878

879

880

881

882

883

884

885

886

887

888

889

890

891

892

893

894

895

896

897

898

899

900

901

902

903

904

905

906

907

908

909

910

911

912

913

914

915

916

917

918

919

920

Lerner MR, Andrews NC, Miller G, and Steitz JA. 1981. Two small RNAs encoded by Epstein-Barr virus and complexed with protein are precipitated by antibodies from patients with systemic lupus erythematosus. Proc Natl Acad Sci U S A 78:805-809.

Lorenz R, Bernhart SH, Honer Zu Siederdissen C, Tafer H, Flamm C, Stadler PF, and Hofacker IL. 2011. ViennaRNA Package 2.0. Algorithms Mol Biol 6:26. 10.1186/1748-7188-6-26

Massimelli MJ, Kang JG, Majerciak V, Le SY, Liewehr DJ, Steinberg SM, and Zheng ZM. 2011. Stability of a Long Noncoding Viral RNA Depends on a 9-nt Core Element at the RNA 5 ' End to Interact with Viral ORF57 and Cellular PABPC1. International Journal of Biological Sciences 7:1145-1160. DOI 10.7150/ijbs.7.1145

Matera AG, Terns RM, and Terns MP. 2007. Non-coding RNAs: lessons from the small nuclear and small nucleolar RNAs. Nat Rev Mol Cell Biol 8:209-220. 10.1038/nrm2124

Mayr C. 2017. Regulation by 3'-Untranslated Regions. Annu Rev Genet 51:171-194. 10.1146/annurevgenet-120116-024704

McGeoch DJ, Cook S, Dolan A, Jamieson FE, and Telford EA. 1995. Molecular phylogeny and evolutionary timescale for the family of mammalian herpesviruses. $J$ Mol Biol 247:443-458. 10.1006/jmbi.1995.0152

Mitton-Fry RM, DeGregorio SJ, Wang J, Steitz TA, and Steitz JA. 2010. Poly(A) tail recognition by a viral RNA element through assembly of a triple helix. Science 330:1244-1247. 10.1126/science.1195858

Moss WN. 2018a. The ensemble diversity of non-coding RNA structure is lower than random sequence. Noncoding RNA Res 3:100-107. 10.1016/j.ncrna.2018.04.005

Moss WN. 2018b. RNA2DMut: a web tool for the design and analysis of RNA structure mutations. RNA 24:273-286. 10.1261/rna.063933.117

Moss WN, Lee N, Pimienta G, and Steitz JA. 2014. RNA families in Epstein-Barr virus. RNA Biol 11:10-17. $10.4161 /$ rna. 27488

Moss WN, and Steitz JA. 2013. Genome-wide analyses of Epstein-Barr virus reveal conserved RNA structures and a novel stable intronic sequence RNA. BMC Genomics 14:543. 10.1186/14712164-14-543

Nakamura T, Yamada KD, Tomii K, and Katoh K. 2018. Parallelization of MAFFT for large-scale multiple sequence alignments. Bioinformatics 34:2490-2492. 10.1093/bioinformatics/bty121

Nawrocki EP, Burge SW, Bateman A, Daub J, Eberhardt RY, Eddy SR, Floden EW, Gardner PP, Jones TA, Tate J, and Finn RD. 2015. Rfam 12.0: updates to the RNA families database. Nucleic Acids Res 43:D130-137. 10.1093/nar/gku1063

O'Grady T, Cao S, Strong MJ, Concha M, Wang X, Splinter Bondurant S, Adams M, Baddoo M, Srivastav SK, Lin Z, Fewell C, Yin Q, and Flemington EK. 2014. Global bidirectional transcription of the Epstein-Barr virus genome during reactivation. J Virol 88:1604-1616. 10.1128/JVI.02989-13

O'Leary CA, Andrews RJ, Tompkins VS, Chen JL, Childs-Disney JL, Disney MD, and Moss WN. 2019. RNA structural analysis of the MYC mRNA reveals conserved motifs that affect gene expression. PLoS One 14:e0213758. 10.1371/journal.pone.0213758

O'Leary NA, Wright MW, Brister JR, Ciufo S, Haddad D, McVeigh R, Rajput B, Robbertse B, Smith-White B, Ako-Adjei D, Astashyn A, Badretdin A, Bao Y, Blinkova O, Brover V, Chetvernin V, Choi J, Cox E, Ermolaeva O, Farrell CM, Goldfarb T, Gupta T, Haft D, Hatcher E, Hlavina W, Joardar VS, Kodali VK, Li W, Maglott D, Masterson P, McGarvey KM, Murphy MR, O'Neill K, Pujar S, Rangwala SH, Rausch D, Riddick LD, Schoch C, Shkeda A, Storz SS, Sun H, Thibaud-Nissen F, Tolstoy I, Tully RE, Vatsan AR, Wallin C, Webb D, Wu W, Landrum MJ, Kimchi A, Tatusova T, DiCuccio M, Kitts P, Murphy TD, and Pruitt KD. 2016. Reference sequence (RefSeq) database at NCBI: current status, taxonomic expansion, and functional annotation. Nucleic Acids Res 44:D733-745. 10.1093/nar/gkv1189

Peer] reviewing PDF | (2020:04:47551:1:1:NEW 5 Aug 2020) 
921

922

923

924

925

926

927

928

929

930

931

932

933

934

935

936

937

938

939

940

941

942

943

944

945

946

947

948

949

950

951

952

953

954

Pfeffer S, Zavolan M, Grasser FA, Chien M, Russo JJ, Ju J, John B, Enright AJ, Marks D, Sander C, and Tuschl T. 2004. Identification of virus-encoded microRNAs. Science 304:734-736.

10.1126/science.1096781

Smatti MK, Al-Sadeq DW, Ali NH, Pintus G, Abou-Saleh H, and Nasrallah GK. 2018. Epstein-Barr Virus Epidemiology, Serology, and Genetic Variability of LMP-1 Oncogene Among Healthy Population: An Update. Front Oncol 8:211. 10.3389/fonc.2018.00211

Sun R, Lin SF, Gradoville L, and Miller G. 1996. Polyadenylylated nuclear RNA encoded by Kaposi sarcoma-associated herpesvirus. Proc Natl Acad Sci U S A 93:11883-11888. 10.1073/pnas.93.21.11883

Sztuba-Solinska J, Rausch JW, Smith R, Miller JT, Whitby D, and Le Grice SFJ. 2017. Kaposi's sarcomaassociated herpesvirus polyadenylated nuclear RNA: a structural scaffold for nuclear, cytoplasmic and viral proteins. Nucleic Acids Res 45:6805-6821. 10.1093/nar/gkx241

Szymula A, Palermo RD, Bayoumy A, Groves IJ, Ba Abdullah M, Holder B, and White RE. 2018. EpsteinBarr virus nuclear antigen EBNA-LP is essential for transforming naive $B$ cells, and facilitates recruitment of transcription factors to the viral genome. PLoS Pathog 14:e1006890. 10.1371/journal.ppat.1006890

Tompkins VS, Valverde DP, and Moss WN. 2018. Human regulatory proteins associate with non-coding RNAs from the EBV IR1 region. BMC Res Notes 11:139. 10.1186/s13104-018-3250-8

Tunnicliffe RB, Levy C, Ruiz Nivia HD, Sandri-Goldin RM, and Golovanov AP. 2019. Structural identification of conserved RNA binding sites in herpesvirus ORF57 homologs: implications for PAN RNA recognition. Nucleic Acids Res 47:1987-2001. 10.1093/nar/gky1181

Tzellos S, and Farrell PJ. 2012. Epstein-barr virus sequence variation-biology and disease. Pathogens 1:156-174. 10.3390/pathogens1020156

Van Etten J, Schagat TL, and Goldstrohm AC. 2013. A guide to design and optimization of reporter assays for 3' untranslated region mediated regulation of mammalian messenger RNAs. Methods 63:110-118. 10.1016/j.ymeth.2013.04.020

Yamada KD, Tomii K, and Katoh K. 2016. Application of the MAFFT sequence alignment program to large data-reexamination of the usefulness of chained guide trees. Bioinformatics 32:3246-3251. 10.1093/bioinformatics/btw412

Young LS, and Rickinson AB. 2004. Epstein-Barr virus: 40 years on. Nat Rev Cancer 4:757-768. $10.1038 / \mathrm{nrc1452}$

Zeng $\mathrm{Y}$, and Cullen BR. 2003. Sequence requirements for micro RNA processing and function in human cells. RNA 9:112-123. 10.1261/rna.2780503

Peer) reviewing PDF | (2020:04:47551:1:1:NEW 5 Aug 2020) 


\section{Figure 1}

Figure 1. Results for the EBV-BART miRNA cluster.

(a) IGV visualization of results. EBV-1 genome coordinates are followed by ScanFold-Fold predicted base pairs represented as arcs (colored blue and green for bp with average z-score $<-2$ and -1 , respectively, and yellow for bp with negative average $z$-scores $>-1$ ), and the genome sequence ( $A, C, G$, and $T$ are in green, blue, orange and red, respectively). Below this is a bar-graph showing the window z-score values predicted by ScanFold-Scan (each bar sits at the $1^{\text {st }}$ nt of the window and is colored red or blue for negative/positive values, respectively; the range of values are indicated in brackets). A cartoon of the gene structure of EBV-1 is shown in blue with the location of BART miRNAs annotated. (b) At the bottom is the ScanFold-Fold secondary structure model for the EBV-BART3 pre-miRNA hairpin. Mature sequences are annotated in blue and red.

B




\section{Figure 2}

Figure 2. Results for the v-snoRNA1.

(a) IGV visualization of results. EBV-1 genome coordinates are followed by ScanFold-Fold predicted base pairs represented as arcs (colored green for bp with average z-score $<-1$ and yellow for bp with negative average z-scores $>-1)$, the genome sequence $(A, C, G$, and $T$ are in green, blue, orange and red, respectively). (b) To the right is the ScanFold-Fold secondary structure model for v-snoRNA1 where additional sequences, beyond the annotated vsnoRNA1 sequence is colored orange. Proposed $D^{\prime}$ and $D$ interaction sites with host rRNA sequences are indicated in pink and blue, respectively.

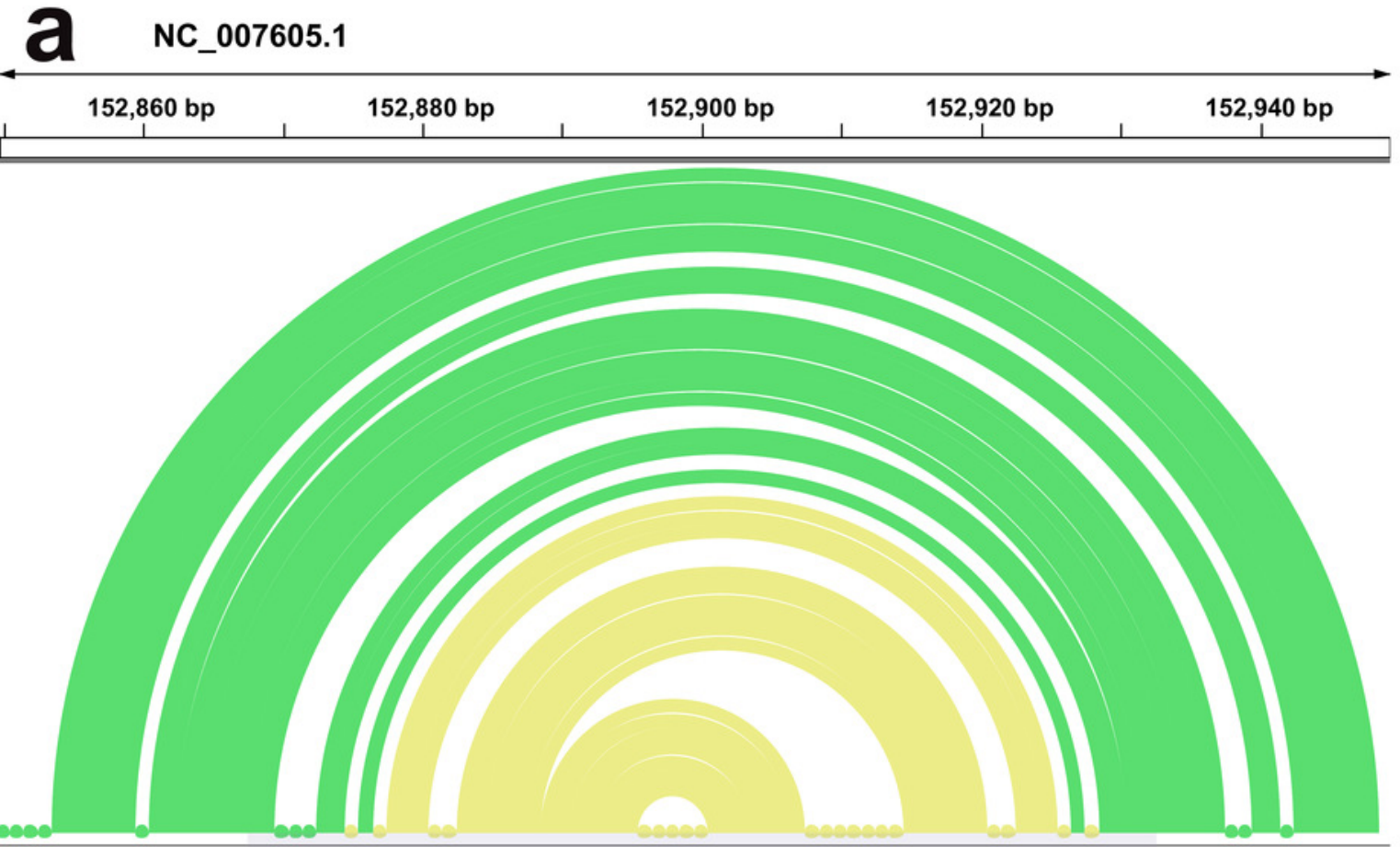


Figure 3

Figure 3. Results for EBER1.

(a) IGV visualization of results. At the top of IGV, EBV-1 genome coordinates are shown, followed by ScanFold-Fold predicted base pairs represented as arcs and the location of EBER1 is indicated by the blue cartoon. Base pairs in the reference EBER1 structure correctly predicted by ScanFold-Fold are highlighted in green on the secondary structure in (b). (c) indicates the correctly predicted pairs after refolding the EBERI sequence using ScanFoldFold bp with average $z$-scores $<-2$ as constraints.

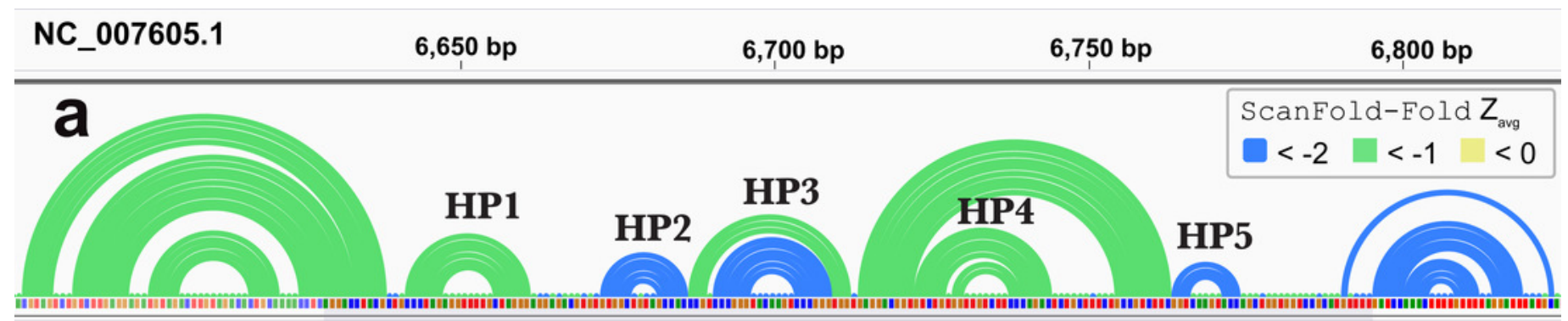

\section{EBER1}
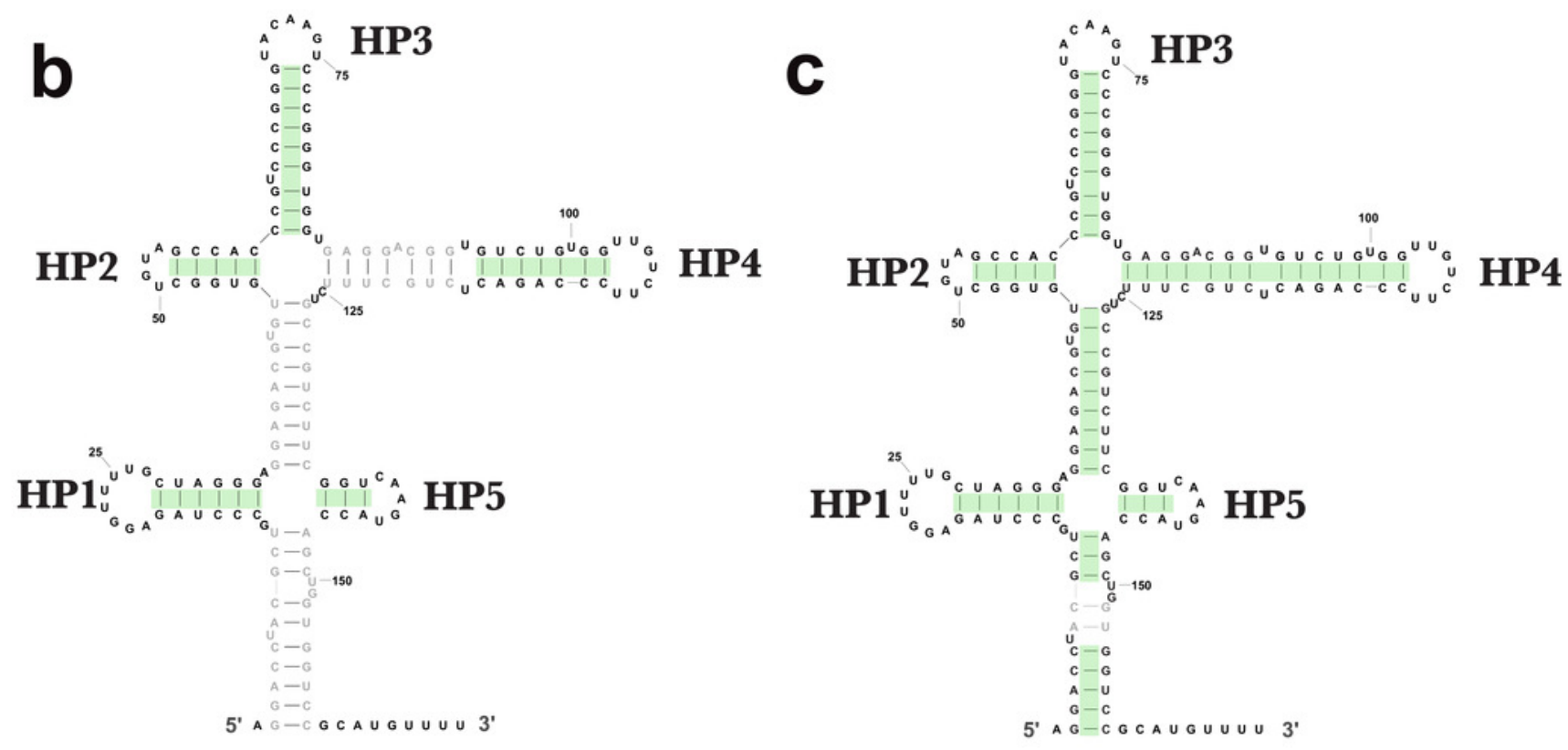


\section{Figure 4}

Figure 4. Results for EBER2 and its interaction sites on the terminal repeat RNA sequence.

(a) EBV-1 genome coordinates are followed by ScanFold-Fold predicted base pairs represented as arcs (colored blue and green for bp with average z-score $<-2$ and -1 , respectively, and yellow for bp with negative average $z$-scores $>-1$ ), the genome sequence ( $A, C, G$, and $T$ are in green, blue, orange and red, respectively). The EBER2 encoding region is indicated with a blue cartoon, this includes an annotation of the terminal repeat (TR) interacting nucleotides in EBER2. (b) ScanFold-Fold predicted base pairs for the TR region, the corresponding binding region to EBER2, represented in panel $A$, with the addition of annotations for the two EBER2 interaction regions (sites A and B) and a bar-graph showing the window $z$-score values predicted by ScanFold-Scan (each bar sits at the $1^{\text {st }} \mathrm{nt}$ of the window and is colored red or blue for negative/positive values, respectively; the range of values are indicated in brackets). (c) Secondary structure model of EBER2 with the TR interacting nucleotides annotated in orange and red for Site A and B, respectively. Model duplexes for each interaction are shown to the right. 


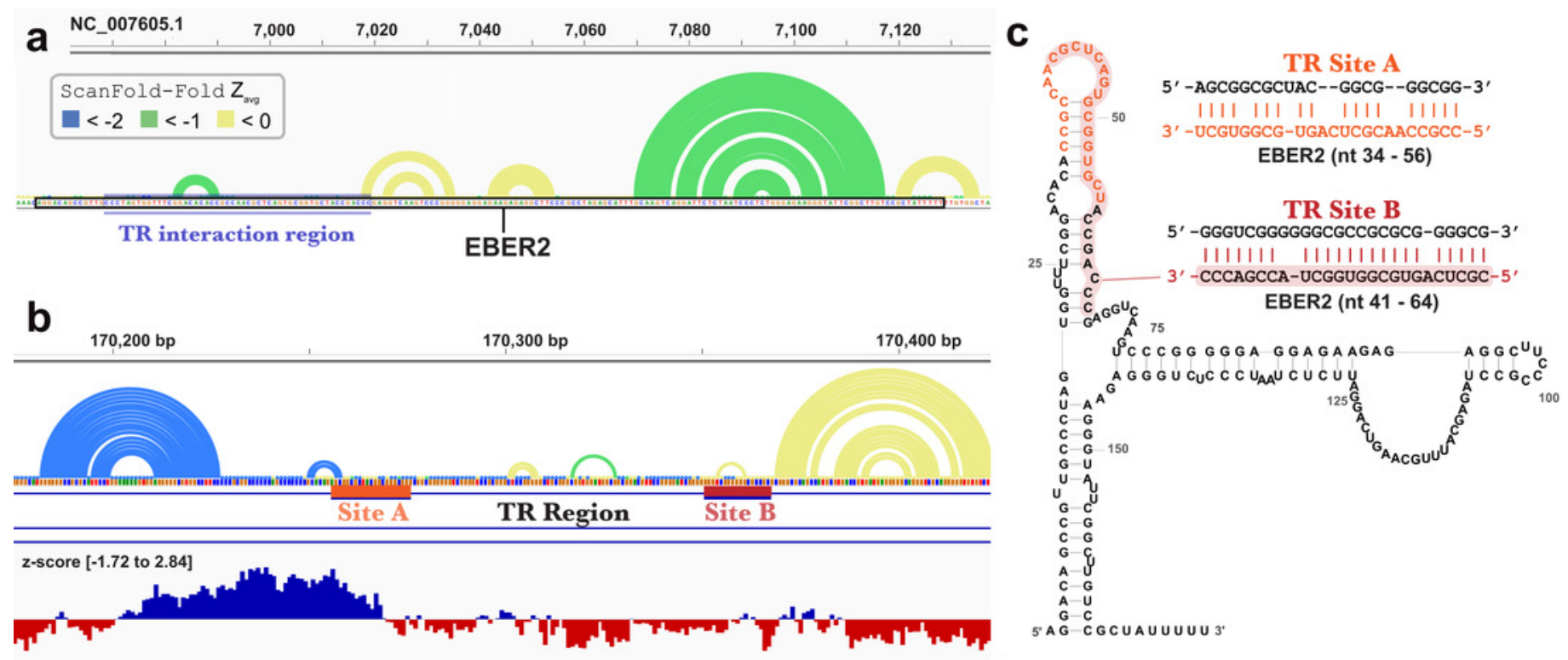


Figure 5

Figure 5. Results for the EBV-1 genome region partially encoding BFRF1-3 and part EBNA1.

EBV-1 genome coordinates are followed by ScanFold-Fold predicted base pairs represented as colored arcs and the location of each gene is indicated by the blue cartoon. The light blue highlighted region is the region used for subsequent experimental analysis.

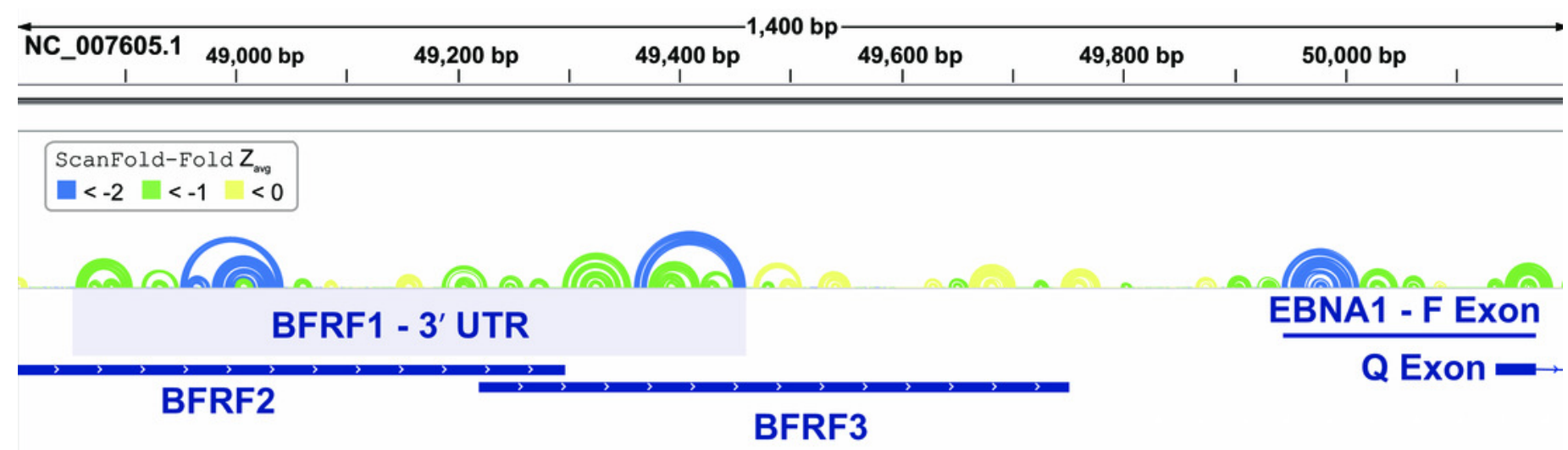




\section{Figure 6}

Figure 6. Results for the region partially overlapping BFRF1-3.

(a) The top of this panel shows ScanFold-Fold predicted base pairs represented as colored arcs. (b) Secondary structure models predicted after energy minimization using ScanFoldFold base pairs (average z-score $<-1$ ) as constraints. Eight motifs (M1-8) were defined (labeled in both the (a) and (b)). Nucleotides with mutations preserving the shown base pair are circled on the structure models. (c) Cartoon showing the locations of three regions (colored green, purple, and red) that were added to downstream of Renilla luciferase (RL; diagrams of constructs are shown with RL in yellow and the added fragments of EBV- 1 in colors corresponding to the cartoon). (d) Results of luciferase assays for each construct; the translational efficiency and luciferase mRNA and protein fold-change (vs. empty vector c ontrol [C]) are plotted for $\mathrm{C}$, w ild t ype (WT) and three fragments of the structured region. The translational efficiencies reported here were calculated by dividing the relative protein abundance of $R L$ (RRR values) by mRNA levels of $R L\left(2^{-\triangle D C T}\right.$ values); see methods for detail and Table S2 for raw values.

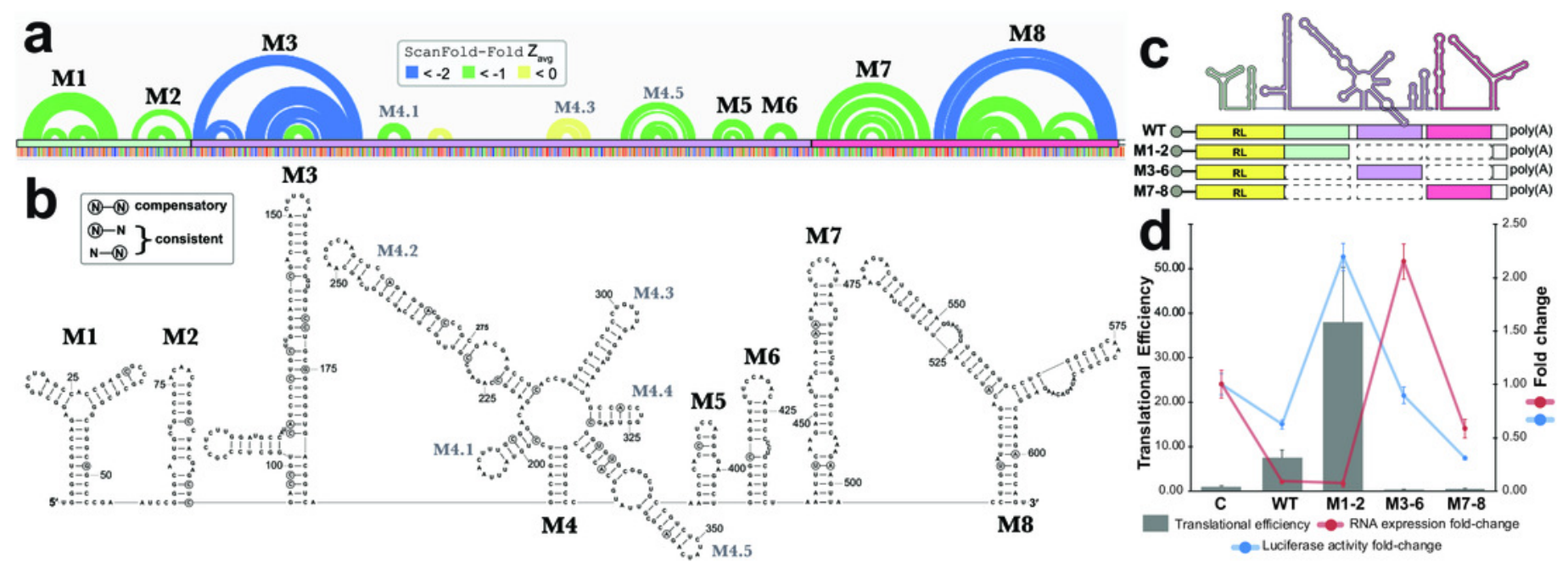




\section{Figure 7}

Figure 7. Results for the EBNA1 5' UTR.

(a) Cartoon showing the EBV-1 genome region with the Fp (used in lytic replication) and Qp (used in latency type I and II) promoters annotated alongside the F/Q, U, and K exons (with start codon colored green).(b) ScanFold-Fold predicted base pairs represented as colored arcs above the sequence of the EBNA1 5' UTR formed after transcription from Fp. Spliced exon sequences are highlighted and labeled below the arc diagram structure (with highlights colored as in the cartoon). (c) Secondary structure model of the EBNA1 5' UTR based on energy minimization using ScanFold-Fold base pairs with average $z$-scores $<-1$ as constraints. The locations of exon starts and the start codon are annotated; as well, nt that show evidence of undergoing structure-preserving mutations are circled. 


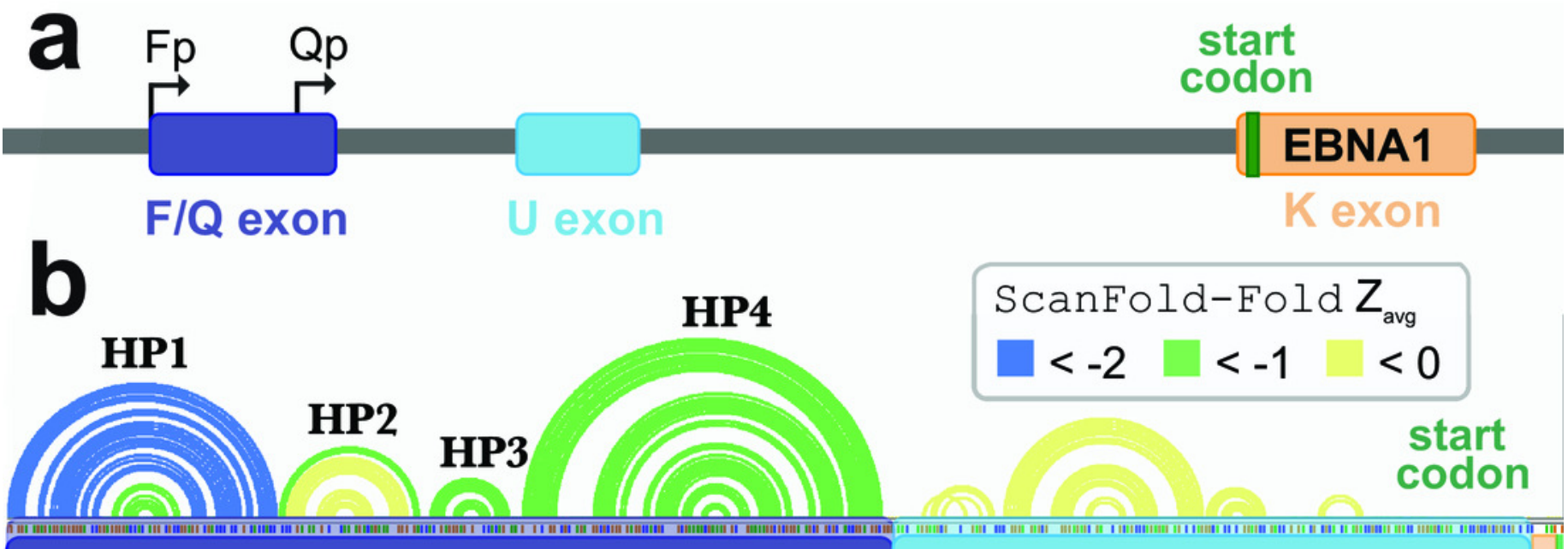

\section{F/Q exon}

C

HP1

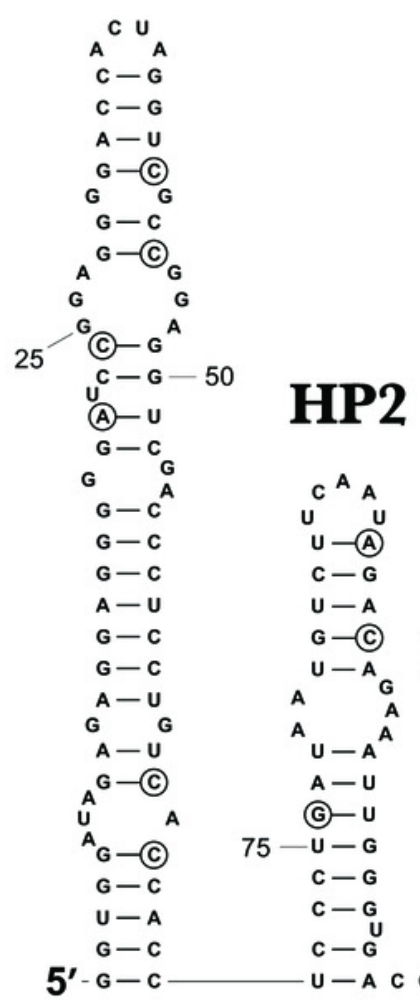

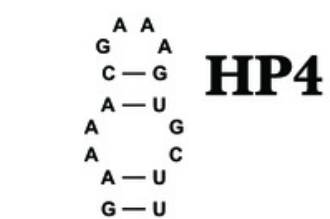

$175-\mathbf{G}-\mathbf{U}$

G ${ }_{U}^{-A_{A}^{A}}$

$U-A$

(1)- $G$

(C)- $G$

(C)- $-\mathrm{G}-200$

(C) $-G$

$a^{C-G}$

$\stackrel{G}{G}_{G^{A}}^{G^{C-G}-G^{A}}{ }^{A}$

a-c)

$G-u^{G}$

(c)-(a)

G-(C)

$\mathbf{C}-\mathbf{G}$

150

${ }^{G}-U$

HP3 3

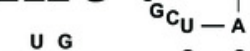

$\begin{array}{ll}G^{U} \mathrm{G} & \mathrm{G}-\mathrm{C}\end{array}$

G-C

$U-G$

$\begin{array}{lr}G-C-125 & C-G \\ A & \text { U }\end{array}$

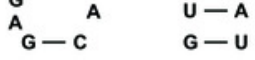

$U-A \quad U-G$

$C-G \quad$ (D) $-G-225$
J exon

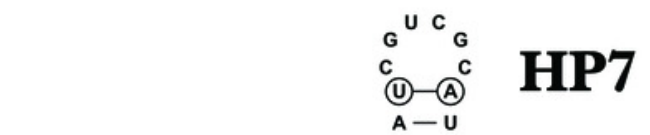

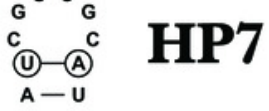

G-C

$U-A$

$\begin{array}{ll}G-U \\ U-A & 350\end{array}$

$U-A$
$C-G$

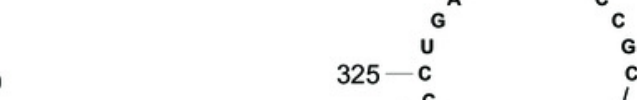

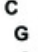

HP6

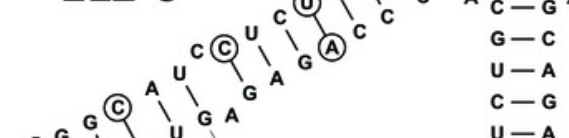

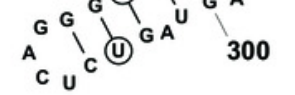

HP5

${ }_{G}^{C G}{ }^{C}$

A-U

$G-C$

$G-U$

A-U

(1)-G-250

$U-A$

A $^{\mathrm{C}-\mathrm{G}} \mathrm{C}$

${ }_{U}^{A} \quad$ U

$G-C$

$G-C$ U
(N-) (N) compensatory

(N) $-\mathrm{N}\}$ consistent

$G$

u

$u-G^{c}$

$G-C$

$c-G$

A $C$

$G-C$
$U-G$

$A-U$

$G-C$

$\begin{array}{lll}\text { G-C } & 400 \\ \text { G-U } & \text { COdOn } \\ \text { G-C C U U U A A G U G U G A A U C A U G } & \end{array}$ 


\section{Figure 8}

Figure 8. Results for KSHV PAN.

(a) IGV visualization of results with KSHV genome coordinates followed by ScanFold-Fold predicted base pairs represented as arcs (colored green for bp with average z-score $<-1$, yellow for bp with negative average $z$-scores $>-1$, and grey for $>0$ ), the genome sequence ( $A, C, G$, and $T$ are in green, blue, orange and red, respectively). (b) The bottom panel contains motif secondary structures predicted by ScanFold-Fold depicted using VARNA (Darty et al. 2009) . The MRE is represented as an orange highlight on the arc diagrams and as orange bps in the secondary structures. The nucleotides from the ENE motif which have been crystalized (Mitton-Fry et al. 2010) are highlighted in green.

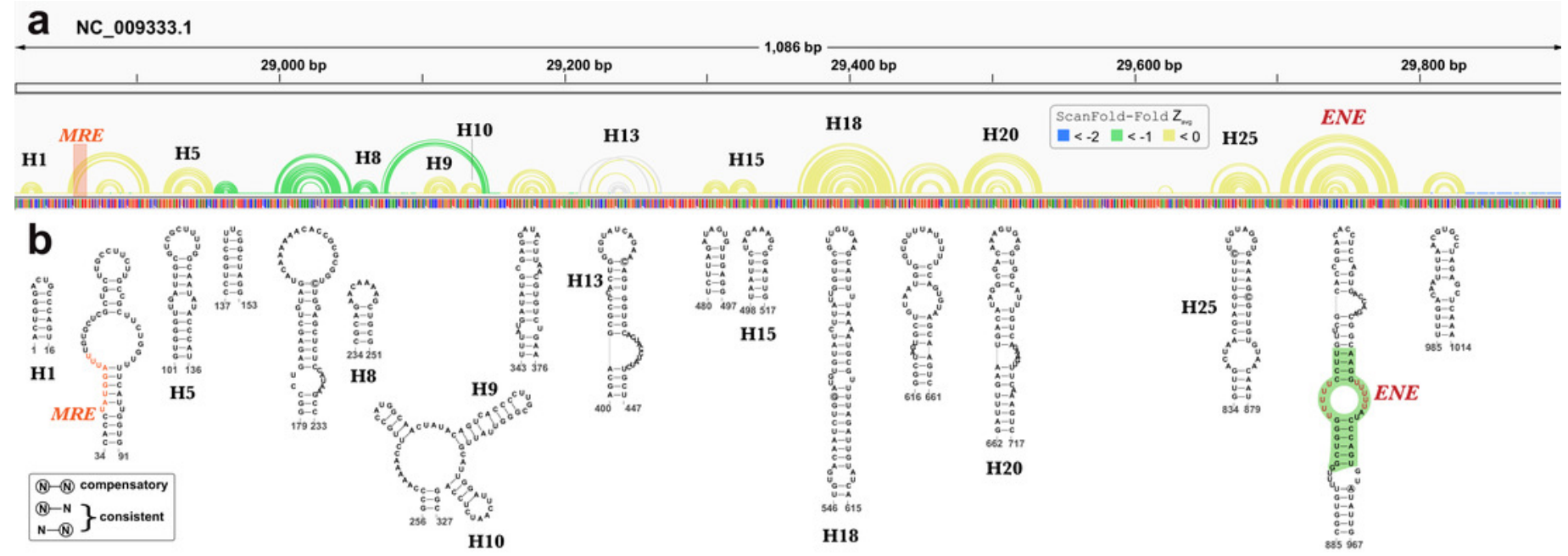




\section{Table $\mathbf{1}$ (on next page)}

Table 1. ScanFold metrics of the human herpesviruses.

ED (ensemble diversity) is a measure of conformational diversity of RNA. Average Genome ZS (z-score) gives the $\Delta \mathrm{G}$ z-score averages across all windows spanning each genome. $\mathrm{ZS}<$ -1 and $<-2$ give the percentage of predictions windows with $\Delta G$ z-score below each cutoff. Total base pairs gives the total number of stable pairs predicted in each genome (counting both strands and all potential base pairs per nucleotide) and the bp ZS $<-1$ and $<-2$ give the percentages of bp below each z-score threshold. Motifs reports the number of individual discrete RNA structures (defined as having a single base helix) containing at least one bp with ZS $<-2$. 


\begin{tabular}{|c|c|c|c|c|c|c|c|c|c|c|c|}
\hline Virus & GC\% & $\underset{(\mathrm{kcal} / \mathrm{mol})}{\Delta \mathrm{G}}$ & ED & $\begin{array}{c}\text { Averag } \\
\text { e ZS }\end{array}$ & $\begin{array}{c}\text { Window } \\
\mathrm{s}\end{array}$ & $\begin{array}{c}Z S<- \\
1\end{array}$ & $\begin{array}{c}Z S<- \\
2\end{array}$ & Total bp & $\begin{array}{c}\text { bp } Z S<- \\
1\end{array}$ & $\begin{array}{c}\mathrm{bp} Z \mathrm{ZS}<- \\
2\end{array}$ & $\begin{array}{l}\text { Motif } \\
\mathrm{s}\end{array}$ \\
\hline EBV-1 & $\begin{array}{c}59.4 \\
\% \\
59.6\end{array}$ & -50.1 & $\begin{array}{c}32 . \\
\mathbf{2} \\
32 .\end{array}$ & -0.63 & 343,348 & $35.1 \%$ & $13.2 \%$ & 835,324 & $8.8 \%$ & $2.5 \%$ & 858 \\
\hline EBV-2 & $\begin{array}{c}\% \\
53.7\end{array}$ & -50.3 & $\begin{array}{c}3 \\
32 .\end{array}$ & -0.62 & 345,230 & $34.4 \%$ & $12.7 \%$ & 850,827 & $8.4 \%$ & $2.6 \%$ & 870 \\
\hline KSHV & $\begin{array}{c}\% \\
68.3\end{array}$ & -42.6 & $\begin{array}{c}6 \\
32 .\end{array}$ & -0.31 & 275,640 & $25.0 \%$ & $7.9 \%$ & 628,314 & $6.4 \%$ & $1.5 \%$ & 381 \\
\hline HSV-1 & $\begin{array}{c}\% \\
36.2\end{array}$ & -60.8 & $\begin{array}{c}7 \\
33 .\end{array}$ & -0.26 & 304,146 & $24.4 \%$ & $8.1 \%$ & 856,374 & $5.4 \%$ & $1.4 \%$ & 436 \\
\hline HHV-7 & $\begin{array}{c}\% \\
70.4\end{array}$ & -24.8 & $\begin{array}{c}7 \\
32 .\end{array}$ & -0.26 & 305,862 & $22.8 \%$ & $7.6 \%$ & 536,275 & $6.8 \%$ & $1.6 \%$ & 320 \\
\hline HSV-2 & $\begin{array}{c}\% \\
57.4\end{array}$ & -64.1 & $\begin{array}{c}7 \\
32 .\end{array}$ & -0.20 & 309,052 & $22.8 \%$ & $7.5 \%$ & $\begin{array}{l}905,657 \\
\mathbf{1}, \mathbf{1 4 3 , 9 7}\end{array}$ & $5.0 \%$ & $1.2 \%$ & 648 \\
\hline HCMV & $\begin{array}{c}\% \\
46.0\end{array}$ & -46.8 & $\begin{array}{c}9 \\
32 .\end{array}$ & -0.19 & 470,994 & $22.9 \%$ & $6.9 \%$ & 3 & $2.9 \%$ & $0.6 \%$ & 562 \\
\hline $\begin{array}{l}\text { VZV } \\
\text { HHV- }\end{array}$ & $\begin{array}{c}\% \\
42.4\end{array}$ & -34.5 & $\begin{array}{c}9 \\
32 .\end{array}$ & -0.13 & 249,470 & $20.0 \%$ & $5.1 \%$ & 498,533 & $5.7 \%$ & $1.0 \%$ & 226 \\
\hline $\begin{array}{c}6 \mathrm{~A} \\
\mathrm{HHV}-\end{array}$ & $\begin{array}{c}\% \\
42.8\end{array}$ & -29.7 & $\begin{array}{c}7 \\
32 .\end{array}$ & -0.12 & 318,458 & $19.6 \%$ & $5.4 \%$ & 597,872 & $5.9 \%$ & $1.2 \%$ & 274 \\
\hline $6 \mathrm{~B}$ & $\%$ & -29.7 & 9 & -0.09 & 323,930 & $19.0 \%$ & $4.7 \%$ & 607,730 & $5.8 \%$ & $0.8 \%$ & 268 \\
\hline
\end{tabular}

1

Table 1. ScanFold metrics of the human herpesviruses. ED (ensemble diversity) is a measure of conformational diversity of RNA. Average Genome ZS (z-score) gives the $\Delta \mathrm{G}$ z-score averages across all windows spanning each genome. $Z S<-1$ and $<-2$ give the percentage of predictions windows with $\Delta G$ z-score below each cutoff. Total base pairs gives the total number of stable pairs predicted in each genome (counting both strands and all potential base pairs per nucleotide) and the bp ZS $<-1$ and $<-2$ give the percentages of bp below each z-score threshold. Motifs reports the number of individual discrete RNA structures (defined as having a single base helix) containing at least one bp with ZS <-2. 